\title{
Numerical Simulation of Natural Convection in Heterogeneous Porous media for $\mathrm{CO}_{2}$ Geological Storage
}

\author{
Panneerselvam Ranganathan • Rouhollah Farajzadeh • \\ Hans Bruining • Pacelli L. J. Zitha
}

Received: 16 February 2010 / Accepted: 5 June 2012 / Published online: 10 August 2012

(C) The Author(s) 2012. This article is published with open access at Springerlink.com

\begin{abstract}
We report a modeling and numerical simulation study of density-driven natural convection during geological $\mathrm{CO}_{2}$ storage in heterogeneous formations. We consider an aquifer or depleted oilfield overlain by gaseous $\mathrm{CO}_{2}$, where the water density increases due to $\mathrm{CO}_{2}$ dissolution. The heterogeneity of the aquifer is represented by spatial variations of the permeability, generated using Sequential Gaussian Simulation method. The convective motion of the liquid with dissolved $\mathrm{CO}_{2}$ is investigated. Special attention is paid to instability characteristics of the $\mathrm{CO}_{2}$ concentration profiles, variation of mixing length, and average $\mathrm{CO}_{2}$ mass flux as a function of the heterogeneity characterized by the standard deviation and the correlation length of the log-normal permeability fields. The $\mathrm{CO}_{2}$ concentration profiles show different flow patterns of convective mixing such as gravity fingering, channeling, and dispersive based on the heterogeneity medium of the aquifer. The variation of mixing length with dimensionless time shows three separate regimes such as diffusion, convection, and second diffusion. The average $\mathrm{CO}_{2}$ mass flux at the top boundary decreases quickly at early times then it increases, reaching a constant value at later times for various heterogeneity parameters.
\end{abstract}

Keywords Density-driven natural convection · Porous media $\cdot$ Heterogeneity · $\mathrm{CO}_{2} \cdot$ Solubility $\cdot$ Mass transfer

\author{
List of Symbols \\ Variables \\ A Aspect ratio of the domain $H / L(-)$ \\ $A_{0} \quad$ Amplitude of sinusoidal perturbation \\ c Dimensionless concentration (-) \\ $c^{\prime} \quad$ Concentration $\left(\mathrm{mol} / \mathrm{m}^{3}\right)$
}

P. Ranganathan · R. Farajzadeh · H. Bruining $\cdot$ P. L. J. Zitha $(\varangle)$

Department of Geotechnology, Delft University of Technology, 2628 CN, Delft, The Netherlands e-mail: P.L.J.Zitha@tudelft.nl 


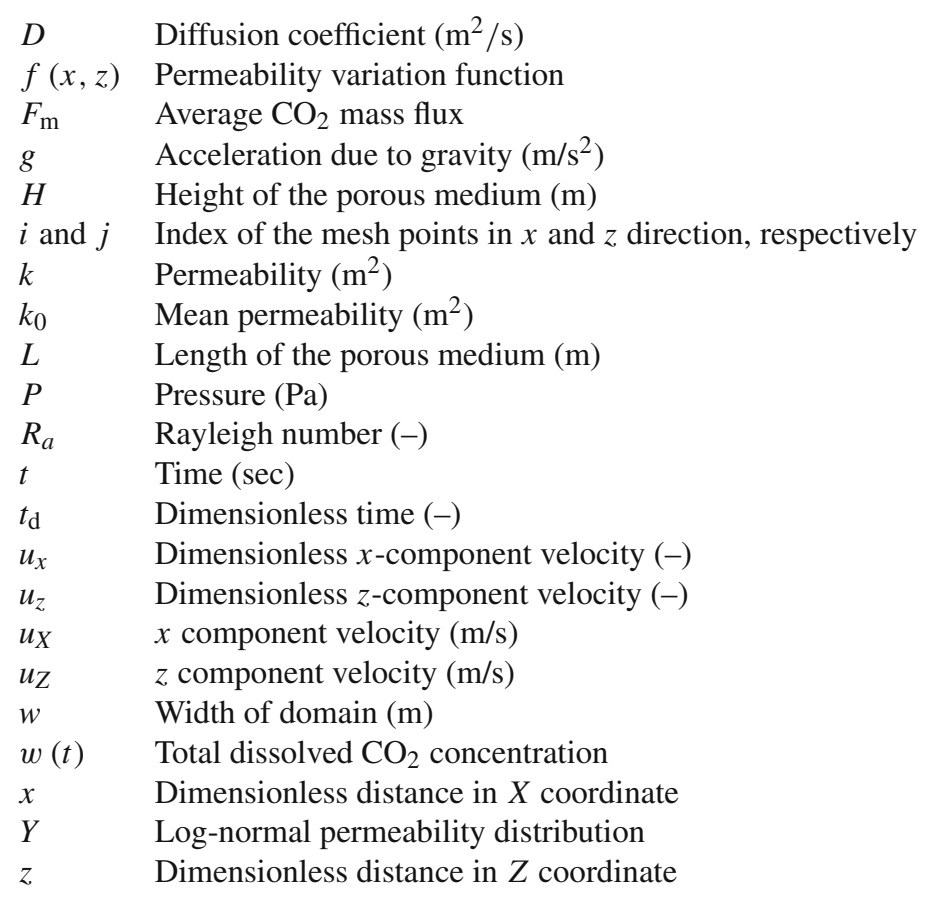

\section{Greek Symbols}

$\alpha \quad$ Wave-number

$\beta_{c} \quad$ The volumetric expansion coefficient

$\varepsilon \quad$ Criteria for convergence

$\varphi \quad$ Porosity (-)

$\lambda_{0} \quad$ Wavelength of sinusoidal perturbation (-)

$\lambda$ Correlation length scale (m)

$\mu \quad$ Viscosity of fluid $(\mathrm{kg} / \mathrm{ms})$

$\theta \quad$ Mean of $\log$ permeability

$\psi \quad$ Stream function $\left(\mathrm{m}^{3} \mathrm{~m}^{-1} \mathrm{~s}^{-1}\right)$

$\rho_{0} \quad$ Pure liquid density $\left(\mathrm{kg} / \mathrm{m}^{3}\right)$

$\rho \quad$ Liquid density $\left(\mathrm{kg} / \mathrm{m}^{3}\right)$

$\Delta \rho \quad$ Change in density $\left(\mathrm{kg} / \mathrm{m}^{3}\right)$

$\sigma \quad$ Standard deviation

$\tau_{x} \quad$ Horizontal correlation length (m)

$\tau_{z} \quad$ Vertical correlation length (m)

\section{Abbreviation}

SGSIM Sequential Gaussian Simulation 


\section{Introduction}

Concern about global warming has increased the impetus to reduce the emissions of $\mathrm{CO}_{2}$, one of the main greenhouse gases, into the atmosphere. The injection of $\mathrm{CO}_{2}$ into aquifers and depleted gas or oil fields is an option for achieving that goal. It can also be used for enhancing gas or oil recovery. Natural convection is one of the main mechanisms to enhance mass transfer of $\mathrm{CO}_{2}$ into the liquid or oil phase during $\mathrm{CO}_{2}$ storage in aquifers and water-flooded oil reservoirs (Firoozabadi and Cheng 2010). The natural convection phenomenon arises because water with dissolved $\mathrm{CO}_{2}$ has a higher density than pure water. Over the last few decades, density-driven natural convection process in porous media received much attention. Many authors (Gebhart et al. 1988; Nield and Bejan 1999; Ennis-King and Paterson 2005; Li et al. 2006; Riaz et al. 2006; Farajzadeh et al. 2007; Hassanzadeh et al. 2007; Pruess and Zhang 2008; Rapaka et al. 2008; Hidalgo and Carrera 2009; Kneafsey and Pruess 2010; Pau et al. 2010) investigated the density-driven natural convection in homogeneous porous media in view of its application to $\mathrm{CO}_{2}$ sequestration. The effect of layering and anisotropy in the permeability fields has also been investigated (Farajzadeh et al. 2008; Rapaka et al. 2009). The convective mixing in a heterogeneous porous medium received less attention in literature compared to the homogeneous case. Nield and Bejan (1999), McKibbin and O'Sullivan (1980), and Malkovsky and Pek (1999) developed analytical solutions for the onset conditions of density-driven natural convection in heterogeneous media for simple geometry and boundary conditions using linear stability analysis. However, this theory does not describe the convective mixing after onset of natural convection. Welty and Gelhar $(1991,1992)$ performed stochastic analyses of one-dimensional density-coupled $\mathrm{CO}_{2}$ transport and derived mathematical expressions for an effective longitudinal, variable-density macrodispersivity. Their analytical expression for the longitudinal macroscopic dispersivity is a function of the geostatistical properties of the porous medium (log-permeability variance and correlation length), concentration gradient, fluid properties, pressure gradient, and time. Schincariol et al. (1997), Simmons et al. (1999, 2001), and Prasad and Simmons (2003) reported numerical modelling studies of natural convection in heterogeneous porous media, with application to groundwater flows. Schincariol et al. (1997) studied the effects of heterogeneity in terms of the variance and the correlation length of the random permeability field on unstable behavior. They reported that longer correlation lengths and higher variance of log-normal permeability distribution promote stability, i.e., less gravity fingering. Simmons et al. (2001) conducted numerical experiments to study variable-density groundwater flows in both periodically structured and random-permeability fields. They found that both the onset and subsequent growth and decay of instabilities are mainly related to the structure and the variance of the permeability field. Prasad and Simmons (2003) used the short heater paradigm (Elder 1967) to study the effect of heterogeneity in permeability distributions on the solute transport by Monte Carlo techniques. They found that an increase in the standard deviation of the log-permeability field results in greater instabilities at earlier times but promotes stability at later times. Further, they found that an increase in the horizontal correlation length of the log-permeability field causes a reduction in the degree of instability. Nield and Simmons (2007) examined the effect of heterogeneity on the onset of natural convection in porous media based on the criterion of "rough-and-ready". Bryant et al. (2008) studied the effect of permeability correlation length and anisotropy on $\mathrm{CO}_{2}$ migration and residual $\mathrm{CO}_{2}$ trapping using single realisation of correlated permeability fields. Recently, Green and Ennis-King (2010) investigated the effect of vertical permeability variations in a reservoir with randomly distributed impermeable barriers on vertical migration of $\mathrm{CO}_{2}$ due to buoyancy using multiple realisations numerical simulation. Nield et al. (2010) investigated the effect of strong heterogeneity (large variation 
of permeability around its mean value) on the onset of convection using spatially correlated log-normal permeability fields and analyzed the result in the statistical framework. Han et al. (2010) investigated the effect of heterogeneity mainly on $\mathrm{CO}_{2}$ trapping mechanism in saline formations. They found that the amount of trapped $\mathrm{CO}_{2}$ depends more on the geometry of the geological structure than on the magnitude of the effective permeability. Recently, we investigated the effect of heterogeneity on density-driven natural convection of $\mathrm{CO}_{2}$ overlying a brine layer (Farajzadeh et al. 2011). The spectral method of Shinozuka and Jan (1972) was used to generate permeability heterogeneity fields. We identified several flow regimes (fingering, channeling, and dispersive) based on the permeability heterogeneity of the medium, i.e., Dykstra-Parsons coefficient $\left(V_{\mathrm{DP}}\right)$ and correlation length. We also found that the rate of mass transfer $\mathrm{CO}_{2}$ into water is higher in heterogeneous media.

This study is a detailed extension of our previous study on the natural convection in heterogeneous media. In the present case, the heterogeneity fields were generated using a more standard Sequential Gaussian Simulation (SGSIM) method. The heterogeneity i.e., the spatial variations of the permeability, is characterized by standard deviation which scales like Dykstra-Parsons coefficient $\left(V_{\mathrm{DP}}\right)$ and the correlation length. The focus here is the effect of the Rayleigh number, evolution of the mixing length, full time dependence of the average $\mathrm{CO}_{2}$ mass flux, effect of boundary conditions and mesh size dependence and the height and width of the domain. This is relevant for $\mathrm{CO}_{2}$ sequestration in aquifers in geologically heterogeneous formations.

The remainder of the paper is organized as follows. Section 2 provides the physical model, the governing equations and the method used to generate the random permeability fields. Section 3 presents the method of solution implemented. Section 4 is devoted to the results and discussion. Section 5 presents the main conclusions of this study.

\section{Model Formulation}

\subsection{Physical Model}

We consider a heterogeneous porous medium with height $H$ and length $L$ (Fig. 1) saturated with water (oil) when a $\mathrm{CO}_{2}$-rich gaseous phase overlays the liquid column. Isothermal conditions are assumed throughout. When $\mathrm{CO}_{2}$ is injected into a saline aquifer, it migrates to the top of the formation due to gravity. Note that our model is also valid for oilfields at the end of the water phase flooding. However, this study is confined to the mixing of $\mathrm{CO}_{2}$ during injection of $\mathrm{CO}_{2}$ into saline aquifers. We consider a subset of the above problem, i.e., a vertical cross section of a single-phase flow aquifer domain, which is overlain by $\mathrm{CO}_{2}$. Recently, Lindeberg and Bergmo (2003) studied numerical simulation of full twophase problem and reported that the interface between the $\mathrm{CO}_{2}$-rich gas and water remains relatively sharp and is not deflected by the fingering in the underlying brine. Ennis-King and Paterson (2003) pointed out that a local equilibrium between the $\mathrm{CO}_{2}$-rich gas and the brine can be assumed at the interface. Accordingly, we assume that interface between the $\mathrm{CO}_{2}$-rich gas and liquid is fixed and sharp, i.e., the capillary forces are neglected. Due to the overlying free $\mathrm{CO}_{2}$ phase, the upper boundary of the single-phase aqueous region remains saturated with $\mathrm{CO}_{2}$. The flow domain is initially in gravity equilibrium (hydrostatic pressure gradient). Furthermore, the flow velocities are small and hence hydrodynamic dispersion reduces to the molecular diffusion (Bear 1972). 


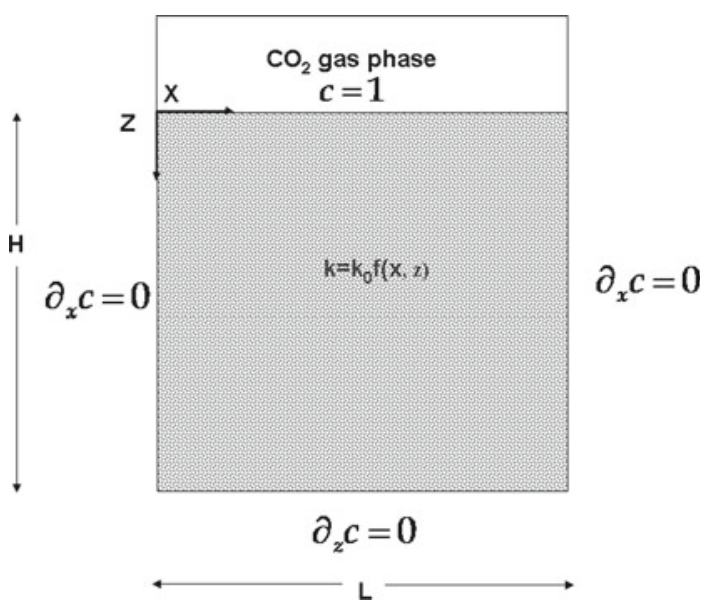

Fig. 1 Schematic representation of present model with initial and boundary conditions

\subsection{Governing Equations}

We consider two-dimensional single-phase flow in a porous medium as depicted in Fig. 1, where the permeability is a function of $x$ and $z$ within the computational domain. We adopt the following notation for the partial derivatives: $\partial_{x} \equiv \partial / \partial x, \partial_{x^{2}}^{2} \equiv \partial^{2} / \partial x^{2}$ for any independent variable $x$. The governing equations can be written as

(a) Continuity equation

$$
\partial_{t} \rho+\partial_{X}\left(\rho u_{X}\right)+\partial_{Z}\left(\rho u_{Z}\right)=0
$$

(b) Darcy's law

$$
\begin{aligned}
& u_{X}=-k_{0} \mu^{-1} f(x, z) \partial_{X} p \\
& u_{Z}=-k_{0} \mu^{-1} f(x, z)\left(\partial_{Z} p-\rho g\right)
\end{aligned}
$$

In these equations $f(x, z)$ describes the permeability variations with respect to mean permeability, $k_{0}$, or otherwise $k(x, z)=k_{0} f(x, z)$.

(c) Concentration

$$
\varphi \partial_{t} c^{\prime}+u_{X} \partial_{X} c^{\prime}+u_{Z} \partial_{Z} c^{\prime}=\varphi D\left(\partial_{X^{2}}^{2} c^{\prime}+\partial_{Z^{2}}^{2} c^{\prime}\right)
$$

The symbols are defined at the start of paper. We use the Boussinesq approximation, which assumes a constant density everywhere in the equation of motion except in the buoyancy term. This approximation is valid since it is assumed here to simplify the mass balance equation, where density variation due to dissolution of $\mathrm{CO}_{2}$ is assumed to be small. The changes of liquid density with the increasing $\mathrm{CO}_{2}$ concentration is assumed to be a liner function of the form

$$
\rho=\rho_{0}+\beta_{c}\left(c^{\prime}-c_{0}^{\prime}\right)
$$

where $\rho_{0}$ is the pure liquid density and $\beta_{c}$ is the coefficient of density increase with concentration. 
Equations (1-4) were solved assuming mainly no-flow boundary conditions at the bottom, left, and right side of the porous medium. The no-flow boundary condition is chosen for the lateral sides of simulation domain to ensure that simulation domain represents a laterally infinity domain. This is a common practice in reservoir simulation studies.

\subsection{Dimensionless Equations}

We take $H$ as the characteristic length and define the following dimensionless variables

$$
x=\frac{X}{H}, \quad z=\frac{Z}{H}, \quad u_{x}=\frac{H}{\varphi D} u_{X}, \quad u_{z}=\frac{H}{\varphi D} u_{Z}, \quad t_{\mathrm{d}}=\frac{D}{H^{2}} t, \quad c=\frac{c^{\prime}-c_{i}^{\prime}}{c_{0}^{\prime}-c_{i}^{\prime}}
$$

and we also define a Rayleigh number, $R_{a}$ by

$$
R_{a}=\frac{\Delta \rho g k_{0} H}{\varphi D \mu}
$$

where $k_{0}$ and $k(x, z)$ are as defined above. Rayleigh Number is dimensionless number that describes instabilities due to natural convection and is defined as the ratio of buoyancy flux to the diffusive flux.

The governing equations then take the form

$$
\begin{aligned}
& \partial_{t_{\mathrm{d}}} \rho+\partial_{x}\left(\rho u_{x}\right)+\partial_{z}\left(\rho u_{z}\right)=0 \\
& u_{x}=-f(x, z) \partial_{x} P \\
& u_{z}=-f(x, z)\left(\partial_{z} P-R_{a} c\right) \\
& \partial_{t_{\mathrm{d}}} c+u_{x} \partial_{x} c+u_{z} \partial_{z} c=\left(\partial_{x^{2}}^{2} c+\partial_{z^{2}}^{2} c\right)
\end{aligned}
$$

we introduce a stream function $\psi$ so that

$$
u_{x}=-\partial_{z} \psi, u_{z}=\partial_{x} \psi,
$$

we also eliminate $P$, thus

$$
\begin{aligned}
& \partial_{x}\left(f^{-1}(x, z) \partial_{x} \psi\right)+\partial_{z}\left(f^{-1}(x, z) \partial_{z} \psi\right)=R_{a} \partial_{x} c \\
& \partial_{t_{\mathrm{d}}} c+\partial_{x} \psi \partial_{z} c-\partial_{z} \psi \partial_{x} c=\left(\partial_{x^{2}}^{2} c+\partial_{z^{2}}^{2} c\right)
\end{aligned}
$$

\subsection{Boundary and Initial Conditions}

The initial condition of the problem is

$$
\psi=0 \text { and } c=0 \text { at } t_{d}=0,
$$

The boundary conditions of the problem are

$$
\begin{aligned}
& \psi=0 \text { and } \partial_{z} c=0 \text { at } x=0, \\
& \psi=0 \text { and } c=1 \text { at } z=0, \\
& \psi=0 \text { and } \partial_{x} c=0 \text { at } z=1, \\
& \psi=0 \text { and } \partial_{x} c=0 \text { at } x=A
\end{aligned}
$$


Table 1 Geostatistical parameters

\begin{tabular}{llll}
\hline Case no. & $\begin{array}{l}\text { Standard } \\
\text { deviation }\end{array}$ & $\begin{array}{l}\text { Correlation } \\
\text { length }\end{array}$ & $\begin{array}{l}\text { Number } \\
\text { realisations }\end{array}$ \\
\hline 1 & 0.1 & 0.025 & 10 \\
2 & 0.1 & 0.05 & 10 \\
3 & 0.1 & 0.1 & 10 \\
4 & 0.1 & 0.25 & 10 \\
5 & 0.3 & 0.025 & 10 \\
6 & 0.3 & 0.05 & 10 \\
7 & 0.3 & 0.1 & 10 \\
8 & 0.3 & 0.25 & 10 \\
9 & 1 & 0.025 & 10 \\
10 & 1 & 0.05 & 10 \\
11 & 1 & 0.1 & 10 \\
12 & 1 & 0.25 & 10 \\
13 & 2 & 0.025 & 10 \\
14 & 2 & 0.05 & 10 \\
15 & 2 & 0.1 & 10 \\
16 & 2 & 0.25 & 10 \\
\hline
\end{tabular}

\subsection{Random Permeability Fields}

We assume that the flow domain represented in Fig. 1 is heterogeneous with a log-normal permeability distribution $[Y=\ln (k)]$. The permeability field is described using the following main parameters: mean permeability, standard deviation, and correlation length in the vertical direction $\tau_{y}$ as well as in the horizontal direction $\tau_{x}$. Its spatial correlation is quantified using exponential autocorrelation function or the variograms (Simmons et al. 2001).

The random permeability fields were generated using Sequential Gaussian Simulation (SGSIM) method developed by GSLib (Deutsch and Journel 1992). SGSIM program is most commonly used to generate random fields of reservoir characterization and is based on multigaussian approach in the spatial distribution of random field. This code generates a standard normal field with a mean of zero and standard deviation of one. We have used the following transformation (Nield et al. 2010) to obtain log-normal distributions which are used as input to our simulation code.

$$
\text { Perm }_{\text {new }}=\exp \left(\theta+\sigma \text { Perm }_{\text {SGSIM }}\right)
$$

In this study, 16 cases of different standard deviations and different correlation lengths are considered. Each case corresponds to a different heterogeneity pattern. The parameters used in each case are shown in Table 1 . The ranges of standard deviation values are similar to values quoted in the literature (LeBlanc et al. 1991; Barlebo et al. 2004).

The flow behavior obtained from a single realisation may be entirely different from the flow pattern for another statically equivalent realisation. To draw any statically significant conclusion, it is necessary to consider a large number of realisations of a single case. In practise, however, this may not be viable as it requires excessive computation time. Since SGSIM code is based on Monte-Carlo simulation for the generation of random field, it can internally, effectively generate multiple equally probable property fields. Thus, a few field 
realisations are used in simulations of density-driven flows (Schincariol et al. 1997; Bryant et al. 2008). For each of the 16 cases, ten realisations of the selected permeability fields were generated.

\section{Method of Solution}

The numerical simulations were done for a domain having a width of $1 \mathrm{~m}$ and a height of 1 $\mathrm{m}$. Such small domain was chosen to insure that we could capture the details of the growth of instabilities due to natural convection. This is required to describe the natural convection behavior at the scale of a grid block, representing a small part of the aquifer. The question whether such a fine scale simulation (grid dimension of $0.01 \mathrm{~m}$ ) is sufficiently accurate will be addressed in Sect. 4.2. The flow domain was discredited in $101 \times 101$ grid cells. A fully implicit finite volume approach (Guceri and Farouk 1985) was used to solve the governing equations, to obtain transient values of the dependent variables in Eq. (14). For each time step, the stream function was computed from Eq. (13) and then concentration profile was obtained by solving Eq. (14). The numerical calculation for each time step was iterated until the following criteria were satisfied

$$
\left|\frac{c_{i, j}^{K+1}-c_{i, j}^{K}}{c_{i, j}^{K+1}}\right|_{\max } \leq \varepsilon \text { and }\left|\frac{\psi_{i, j}^{K}-\psi_{i, j}^{K}}{\psi_{i, j}^{K+1}}\right| \leq \varepsilon
$$

where $i$ and $j$ are the index of the mesh points in $x$ and $z$ direction, respectively, and the superscript $K$ stands for the iteration cycle. For our numerical computations, $\varepsilon$ was set to $10^{-5}$ and the time step was chosen to be $10^{-6}$ to insure accuracy of the results. To observe the fingering behavior it was necessary to disturb the interface. Therefore in the numerical simulations, following the procedure in Farajzadeh et al. (2007), we used sinusoidal initial perturbation are superimposed along with top boundary condition, i.e.,

$$
c(x, z=0, t=0)=1+A_{0} \sin \left(\frac{2 \pi x}{\lambda_{0}}\right)
$$

where $A_{0}$ is the amplitude and $\lambda_{0}$ is the wavelength of sinusoidal perturbation. In this study, $A_{0}=0.01$ and $\lambda_{0}=1 / 12$ is used. We studied the various ranges of wave numbers $\left(\alpha=2 \pi / \lambda_{0}\right)$ between 10 and 270 to investigate the effect of the wavelength on the numerical solution.

\section{Results and discussion}

Below we discuss the instabilities in terms of $\mathrm{CO}_{2}$ concentration profiles, variation of the mixing length, and the average $\mathrm{CO}_{2}$ mass flux at the top boundary. The concentration profiles are expressed as a dimensionless mass fraction of $\mathrm{CO}_{2}$ in the aquifer at any time. The mixing length is defined as the distance from the top boundary to the location where the dimensionless $\mathrm{CO}_{2}$ concentration is equal to 0.1 . The mixing length expresses how the concentration of dissolved $\mathrm{CO}_{2}$ is affected by hydrodynamics. The average $\mathrm{CO}_{2}$ mass flux at the top boundary is calculated using 

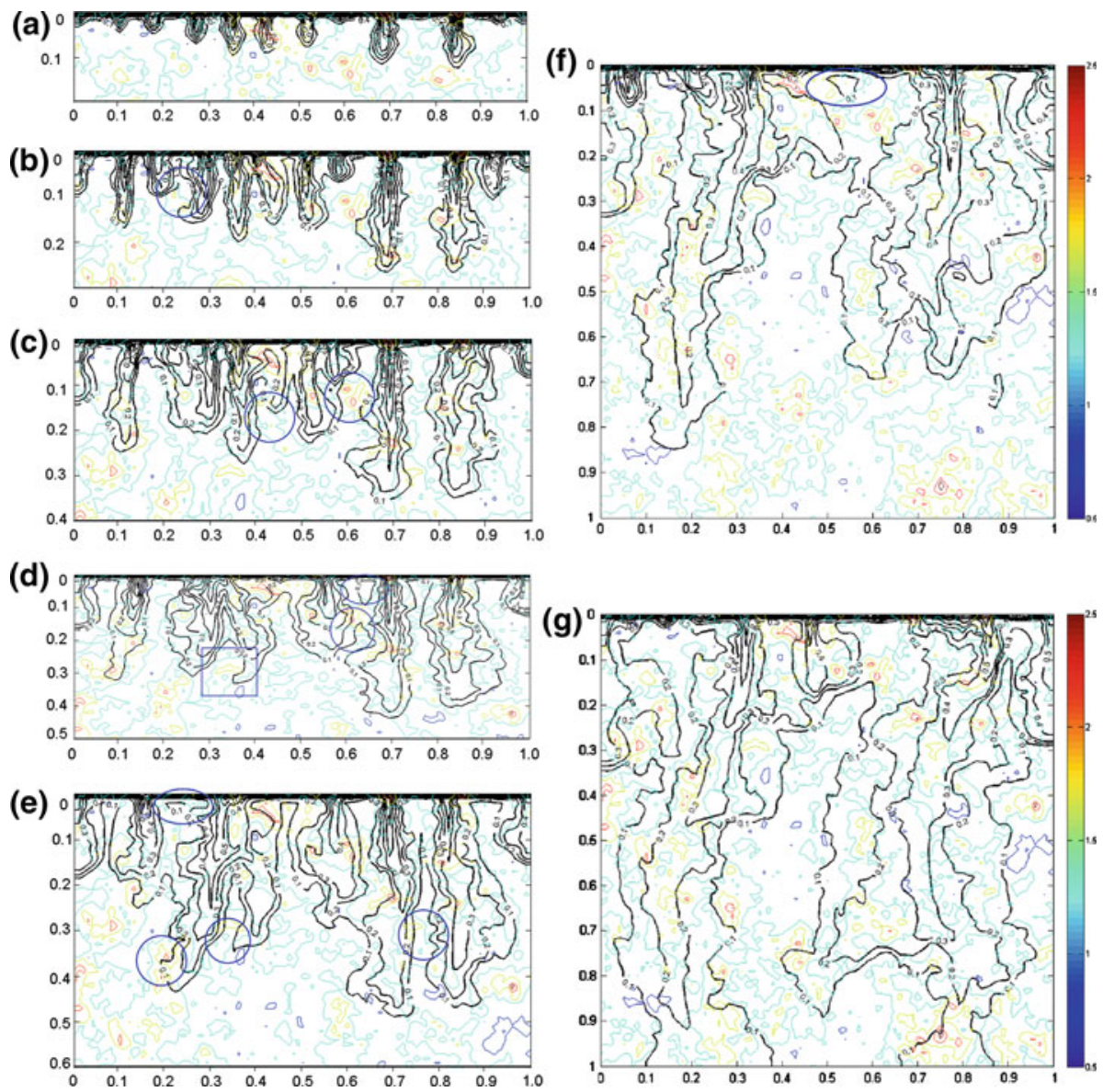

Fig. $2 \mathrm{CO}_{2}$ concentration profiles along with log-normal permeability fields of one realisation $(\sigma=0.3$ and $\lambda=0.1$ ) for Rayleigh number of 10,000. (a) at $t_{d}=0.00005$, (b) at $t_{d}=0.0001$, (c) at $t_{d}=0.00015,(\mathbf{d})$ at $t_{d}=0.0002,(\mathbf{e})$ at $t_{d}=0.00025,(\mathbf{f})$ at $t_{d}=0.0005,(\mathbf{g})$ at $t_{d}=0.00075$

$$
F_{\mathrm{m}}(t)=\int_{0}^{L}\left(\frac{\partial c}{\partial z}\right)_{z=1} \mathrm{~d} x
$$

\subsection{Base Case}

Figure 2 shows the $\mathrm{CO}_{2}$ concentration profiles along with log-normal permeability field at various dimensionless times for a Rayleigh number $R_{a}=10,000$. We generated a single realisation of a log-normal permeability field with a standard deviation of 0.3 and a correlation length of 0.1 in both vertical and horizontal directions. Figure 2 shows that at early times, e.g., 0.00015 (Fig. 2a), the number of fingers is equal to the initial perturbations, 11 ; the fingers grow independently of each other. At longer times, however, the number of fingers is reduced as fingers interact with each other due to various non-linear interaction mechanisms to form a large-scale structure that reaches the bottom of the flow domain. The 


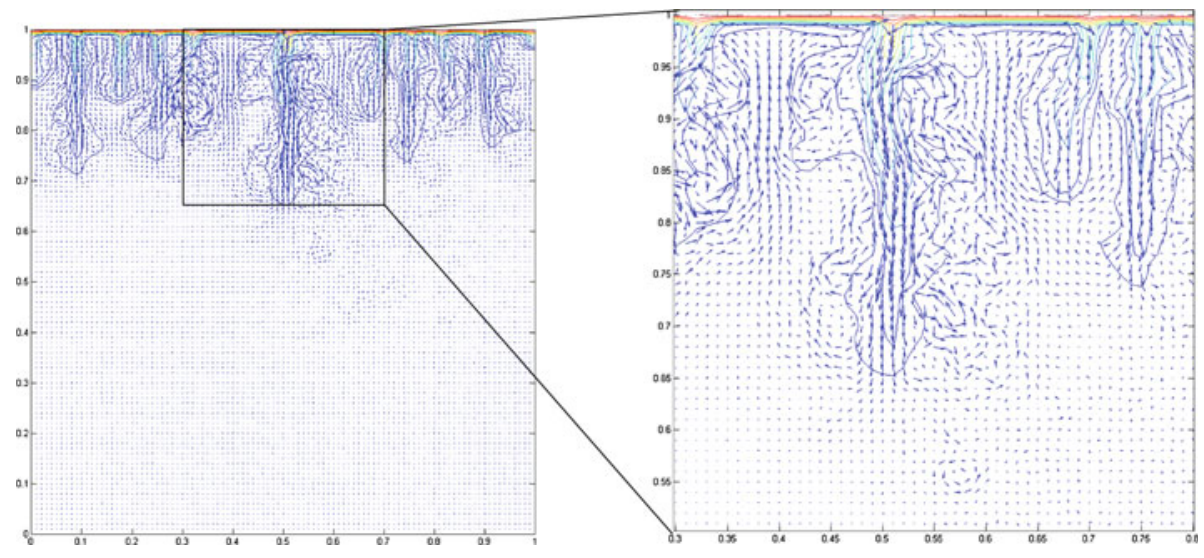

Fig. 3 A snapshot of velocity vectors along with concentration profiles at the dimensionless time of $t_{d}=$ 0.0015 for log-normal permeability field $(\sigma=0.3$ and $\lambda=0.1)$

evolution mechanisms of the fingers include shielding, side-merging, tip splitting, and fading phenomena observed earlier in homogeneous porous medium (Riaz et al. 2006; Farajzadeh et al. 2007). Remarkably, it appears that the interaction of fingers mainly takes place at the high-permeability zones, as shown by the circle in the figures. It is found that the interaction occurs laterally between the dominant fingers and the slowly moving fingers. This mechanism may be referred to as "side merging." The lateral development of the tip of slowly moving fingers that interact with the dominant fingers results in narrowing the neck of the dominant fingers, which is indicated by the square in Fig. 2d. This mechanism may be referred to as "necking." The "side-merging" and "necking" mechanisms were discussed in detail by Zimmerman and Homsy (1992). The other mechanisms of non-linear interaction are tip-splitting and trailing lobe detachment. In tip splitting mechanism, the fingers that move independently get flattened at the tip: this mechanism is not observed in this study. In trailing lobe detachment, a trailing lobe of different concentration forms and detaches itself during the interaction of the fingers. This mechanism is indicated by the ellipse in both Fig. 2e, $\mathrm{f}$. This trailing lobe detachment mechanism was previously reported by Rogerson and Meiburg (1993) and Ghesmat and Azaiez (2008).

Since the velocity plays a major role in the convective mixing, a snapshot of velocity vectors along with the $\mathrm{CO}_{2}$ concentration profiles are plotted in Fig. 3. It is evident from the figures that the circulation pattern can be observed around the fingers tip, whereby the downward velocity vectors (arrows) occur within the fingers and the upward velocity moves toward the top boundary in the region between fingers. It is also observed from the same figures that the velocity is smaller at the regions away from the fingers.

It is interesting to compare how instabilities grow in homogeneous and heterogeneous porous media. Figure 4 shows the concentration profiles obtained for homogeneous media with a Rayleigh number of 10,000. In the homogeneous case, the instabilities grow smoothly due to uniform permeabilities. But in the heterogeneous case (Fig. 2), the instabilities become much more ragged and grow faster than in homogeneous media. The growing instabilities in heterogeneous media exhibit side bumps, i.e., small lateral bulges at the location where the $\mathrm{CO}_{2}$ concentration begins to enter the high permeability regions. In the homogeneous case at later times $\left(t_{d}=0.0005\right)$ fingers merge at the top and split halfway (Fig. 4). In the heterogeneous case, the finger seems to remain separated (Fig. 2). 

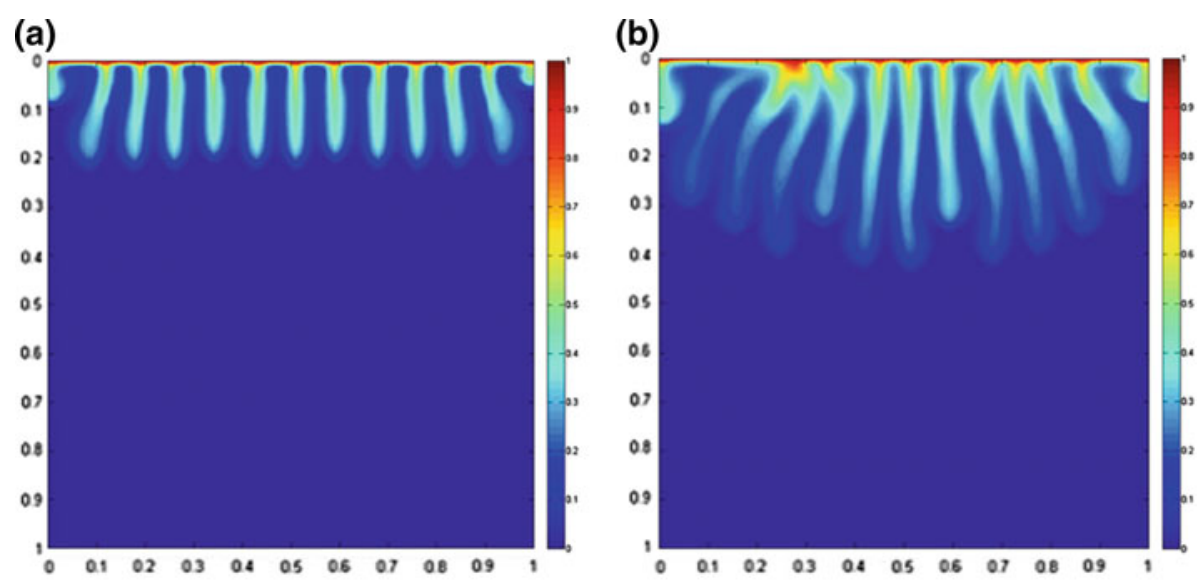

(c)

(d)
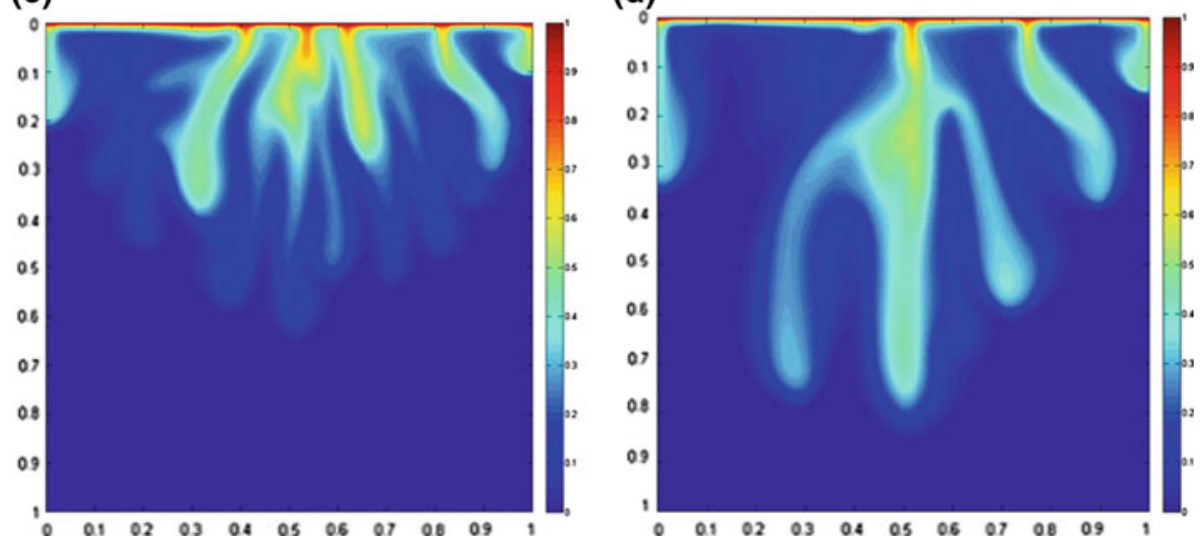

Fig. $4 \mathrm{CO}_{2}$ concentration profiles for Rayleigh number of 10,000 with various dimensionless times for homogeneous porous medium. (a) at $t_{d}=0.00025$, (b) at $t_{d}=0.0005$, (c) at $t_{d}=0.00075$, (d) at $t_{d}=0.001$

\subsection{Effect of Grid Size}

In order to check the effect of grid size, we performed a number of simulations by varying grid size for a particular realisation of the log-normal permeability fields with a standard deviation of 0.3 and a correlation length of 0.1 . Figure 5 shows the plot of the total amount of dissolved $\mathrm{CO}_{2}$ versus dimensionless time for various grid sizes, ranging from 0.008 to $0.0167 \mathrm{~m}$. The total dissolved $\mathrm{CO}_{2}$ concentration is the integral quantity of the dissolution of the $\mathrm{CO}_{2}$ and is given by

$$
w(t)=\int_{0}^{H} \int_{0}^{L} c(x, z, t) \mathrm{d} x \mathrm{~d} z
$$

It is observed that the total dissolved $\mathrm{CO}_{2}$ is same for all grid sizes over time. Note that the simulation with fine grid size $(0.008-0.01 \mathrm{~m})$ predicts slightly lower total dissolved $\mathrm{CO}_{2}$ than predicted using larger grid sizes $(0.0125-0.017 \mathrm{~m})$. Thus, it can be asserted that grid size in the range of $0.008-0.0167 \mathrm{~m}$ is adequate for resolving density-driven mixing. 


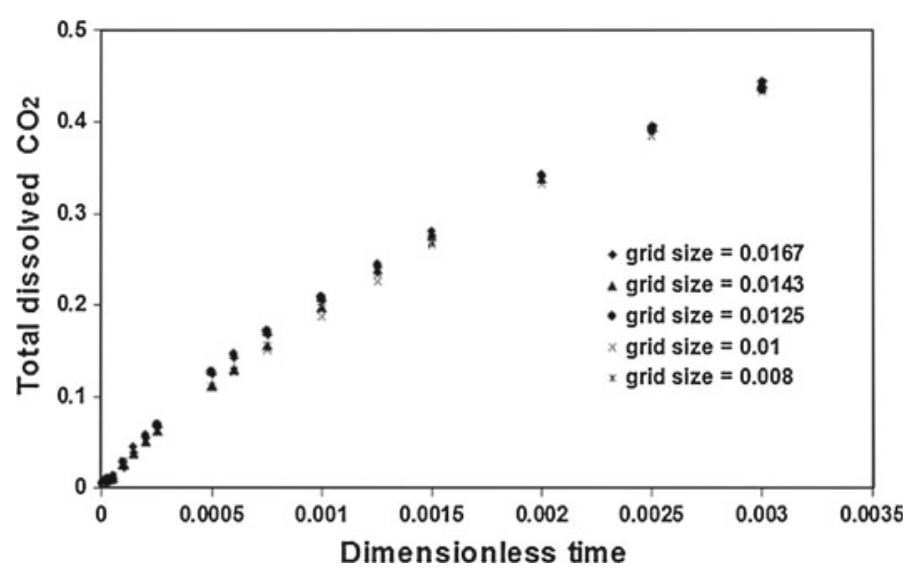

Fig. 5 Effect of mesh sensitivity on the total dissolved $\mathrm{CO}_{2}$

\subsection{Effect of Rayleigh Number}

The effect of varying the Rayleigh number on the $\mathrm{CO}_{2}$ concentration profiles is now examined. Our simulations are based on generated permeability fields leading to Rayleigh numbers of $1000,2000,5000$, and 10,000. The standard deviation and the correlation length in all fields is $\sigma=0.3$ and $\lambda=0.1$. For each condition ten realisations are made. Figure $6 \mathrm{a}$ shows the log-normal permeability field for a single realisation. We first examine the behavior of the instabilities at the early stages. Figure $6 \mathrm{~b}$ shows $\mathrm{CO}_{2}$ concentration profiles for a single realisation for the Rayleigh number $R_{a}=1000$ at $t_{d}=0.001, R_{a}=2000$ at $t_{d}=0.0005, R_{a}=5000$ at $t_{d}=0.00025$, and $R_{a}=10,000$ at $t_{d}=0.0001$. Figures show that the concentration front moves faster as the Rayleigh number increases. For the lower Rayleigh numbers $\left(R_{a}=1000-2000\right)$, there are only a few large fingers, which move independently without interacting with each other. A significant dispersive spreading is also visible in the concentration front. For higher Rayleigh numbers, i.e., (5,000-10,000), the instabilities are more developed and form small-scale complex fingering structures. In addition, the concentration front becomes steeper as the Rayleigh number increases.

Now, we consider the long time behavior of the fingers. The concentration profiles for various Rayleigh numbers at the time that the $\mathrm{CO}_{2}$ plume reaches the bottom boundary are plotted in Fig. 6c. For all values of Rayleigh numbers, it is found that small fingers merge together to form two larger fingers, which reach the bottom of the flow domain. A dispersive spreading in the concentration fronts is also observed for low Rayleigh numbers, whereas the concentration profiles are steeper for high Rayleigh numbers. Fingers grow faster with increasing Rayleigh number.

\subsection{Effect of Standard Deviation}

The effect of standard deviation on the instabilities was investigated using the following values of standard deviations $0.1,0.3,1.0$, and 2 . Figure $7 \mathrm{a}-\mathrm{c}$ shows the concentration profiles and the log-normal permeability field for the values of the standard deviations examined, together 
(a)

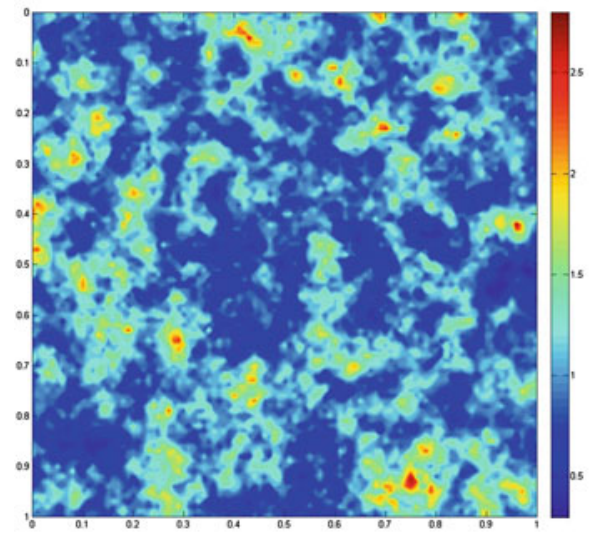

(b)

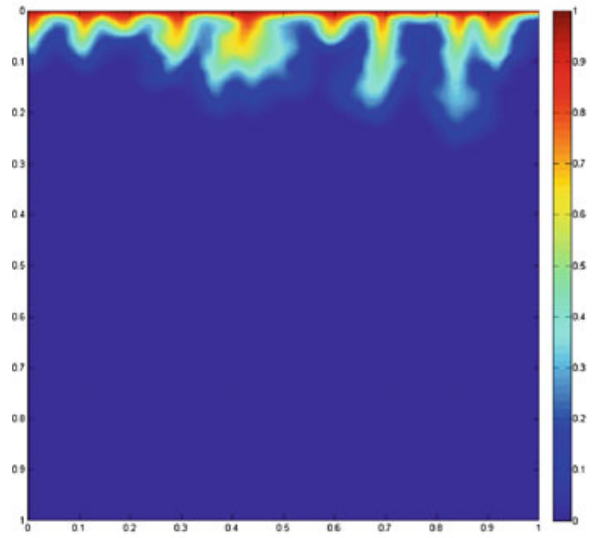

$R_{a}=1000$ at $\tau=0.001$

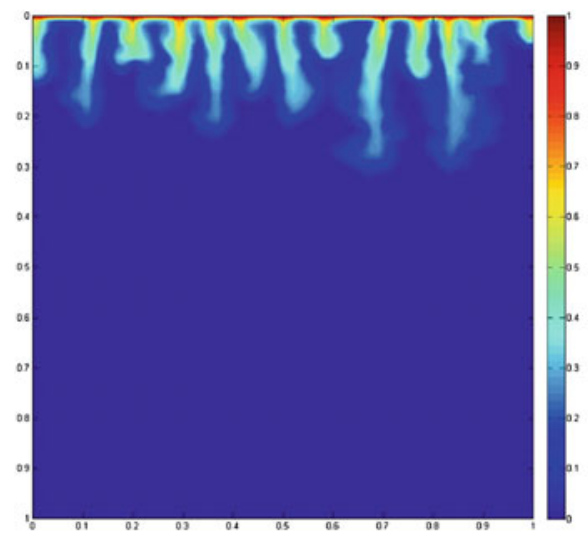

$R_{a}=5000$ at $\tau=0.00025$

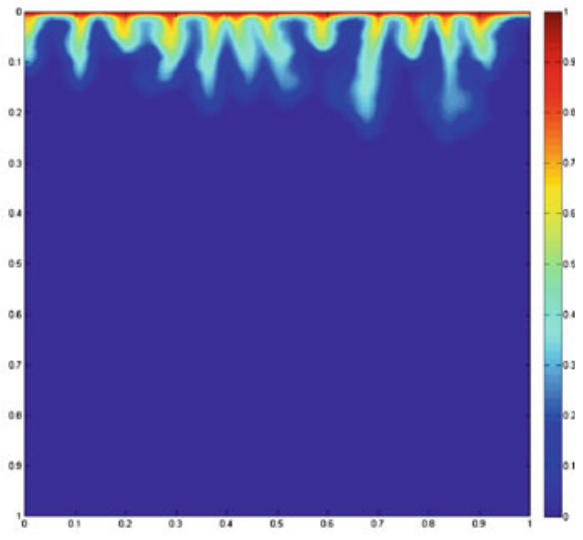

$R_{a}=2000$ at $\tau=0.0005$

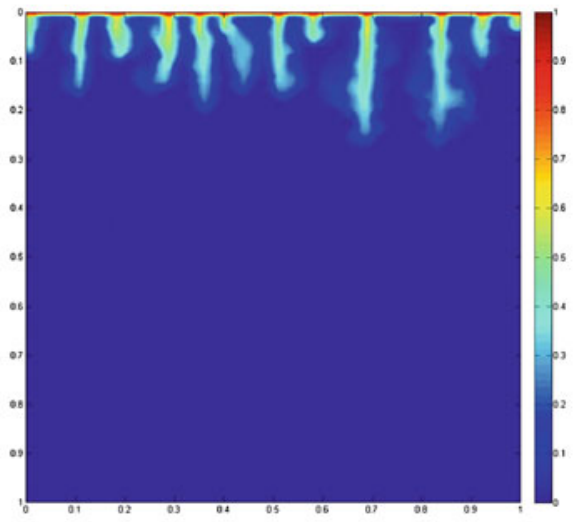

$R_{a}=10000$ at $\tau=0.0001$

Fig. 6 (a) Log-normal permeability field of one realisation, (b) $\mathrm{CO}_{2}$ concentration profiles at earlier time, (c) at later time for various Rayleigh numbers near the time at which a finger hits the bottom 
(c)
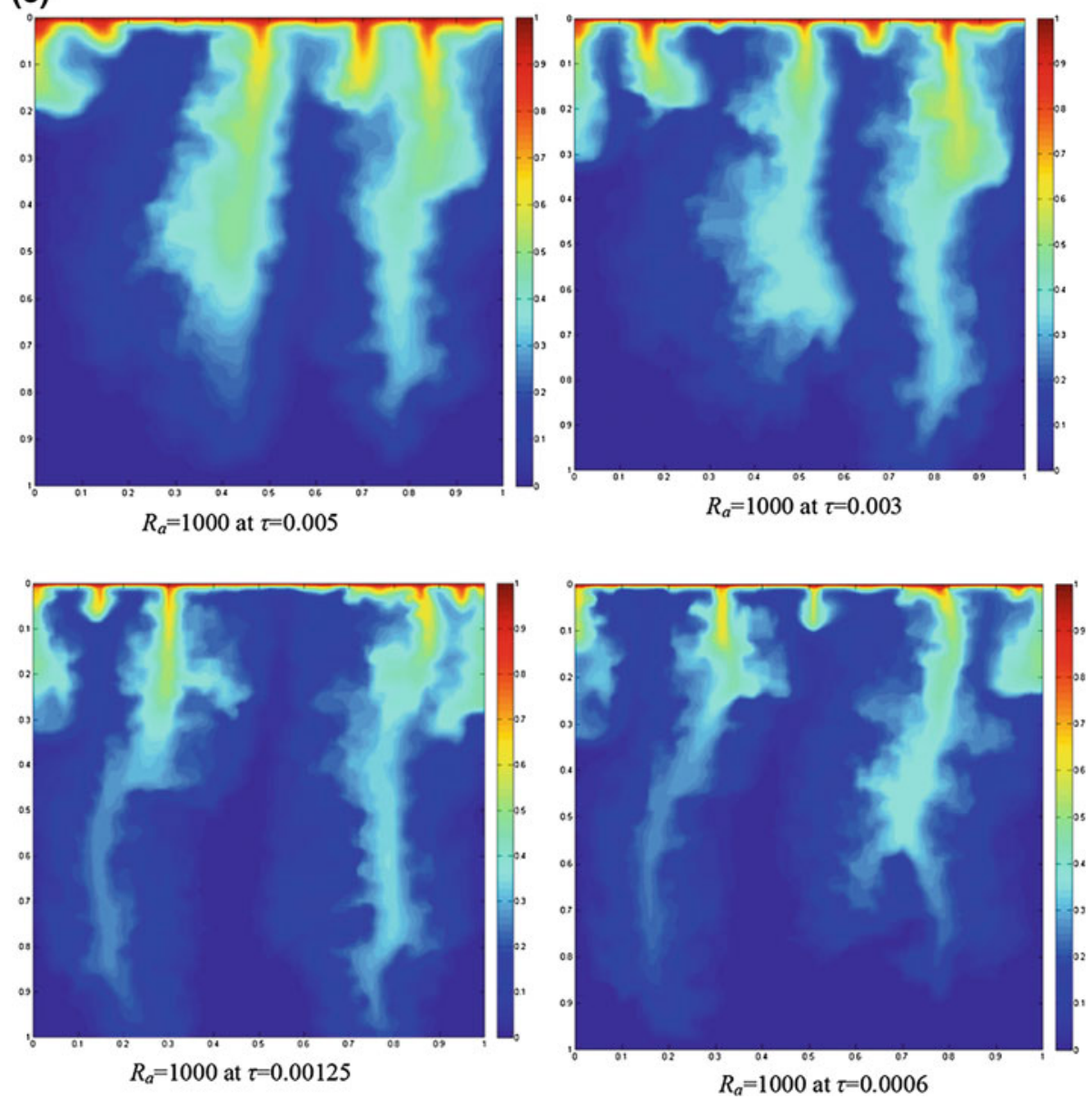

Fig. 6 continued

with different correlation length at a dimensionless time of 0.0005 . At very small standard deviations $(\sigma \rightarrow 0)$, i.e., for small degrees of heterogeneity, the instability behavior is similar to the homogeneous case (Farajzadeh et al. 2007). As $\sigma$ increases, there is a significant change in the concentration profiles, as can be seen from Fig. 7. For lower standard deviation $(\sigma=0.3)$, the estimated average logarithm of the permeability field obtained from a SGSIM simulation varies between 0.7 and 1.4. For higher standard deviation, the estimated logpermeability field varies from 500 to 3500 . In the literature, the behavior of instabilities defined was categorized into fingering, channeling, and dispersive behavior (Waggoner et al. 1992; Sorbie et al. 1994; Farajzadeh et al. 2011). The fingering pattern means that instabilities grow along the preferential flow path, which is formed by permeability variations. Channeling behavior of the instabilities show that instabilities move toward the high permeable zone and are controlled by the permeability distribution pattern. The dispersive flow pattern of instabilities occurs when the length scale of instabilities is smaller than the correlation length scale of the permeability field and is characterized by the square root of time. 
(a)

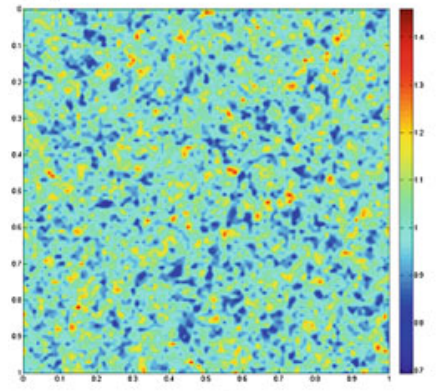

$\sigma 0.1$

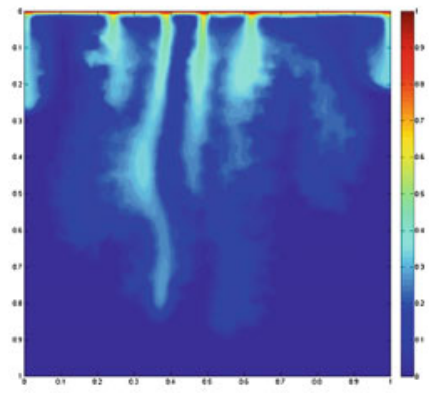

Fingering
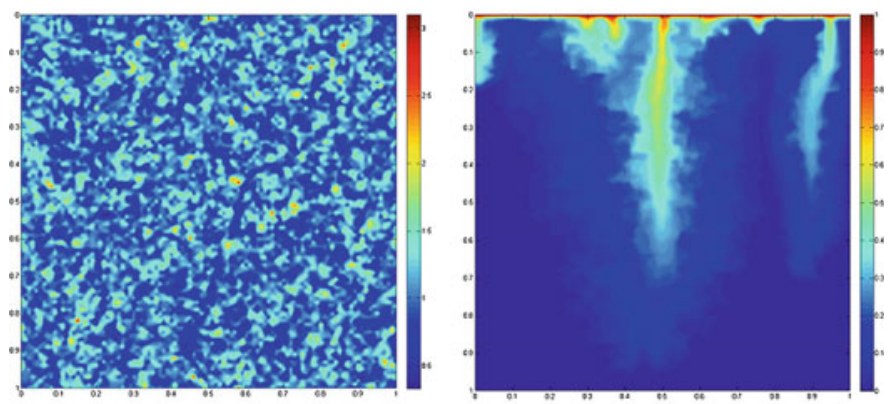

$\sigma=0.3$

Channelling
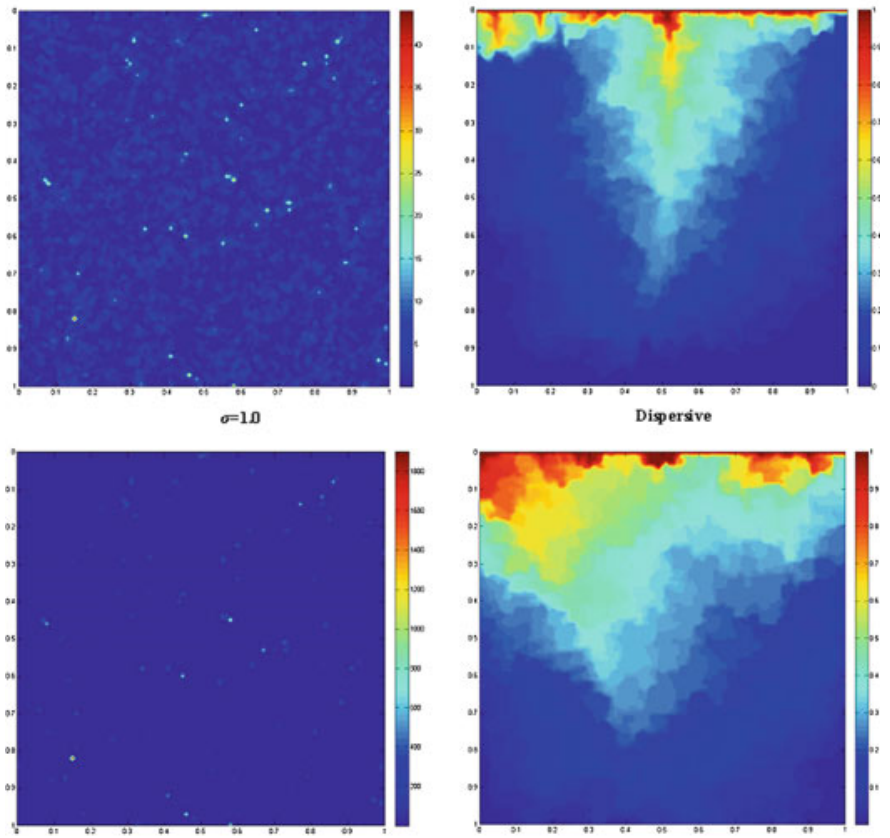

$\sigma=20$

Dispersive

Fig. 7 Log-normal permeability fields and $\mathrm{CO}_{2}$ concentration profiles of one realisation for Rayleigh number of 10,000 at dimensionless time $t_{d}=0.0005$ with different standard deviations for correlation lengths of (a) 0.025 , (b) 0.1 , (c) 0.25 
(b)
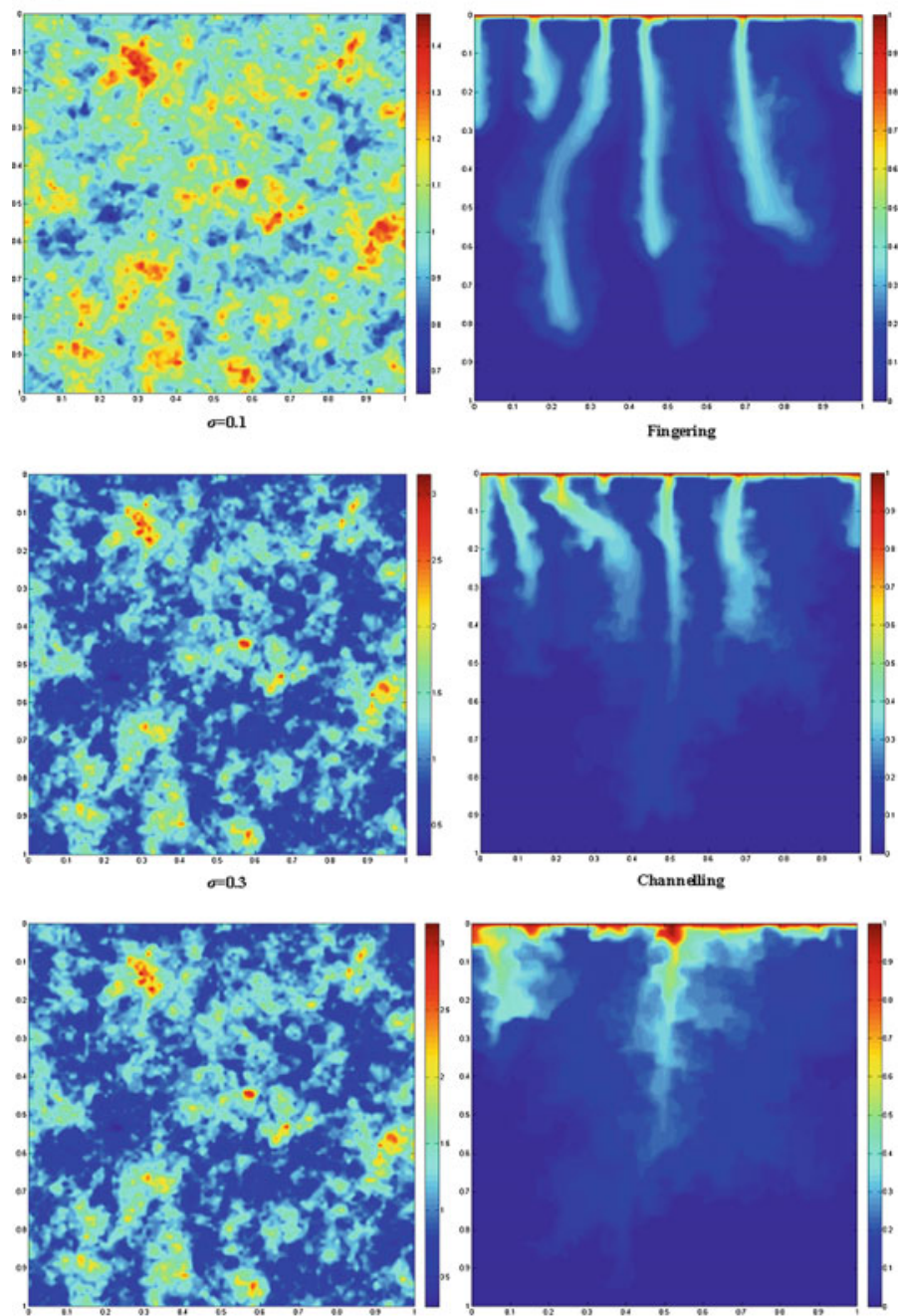

$\sigma=10$
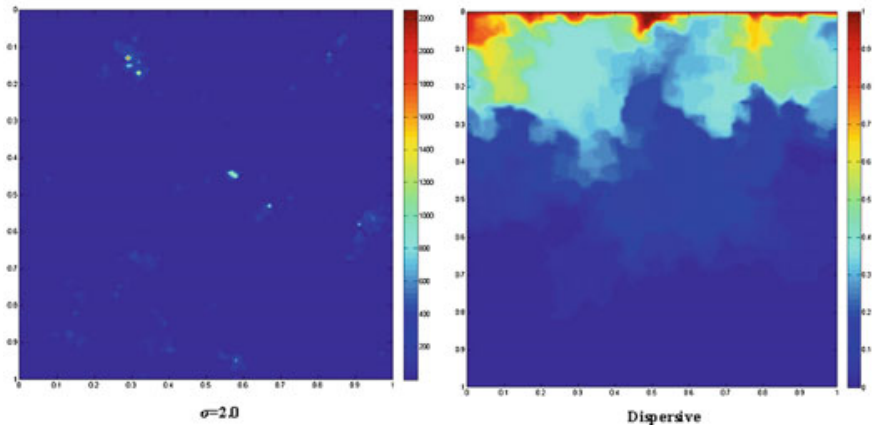

Fig. 7 continued 


\section{(c)}

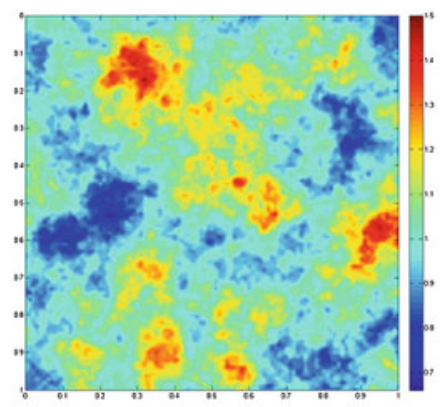

$\sigma=0.1$
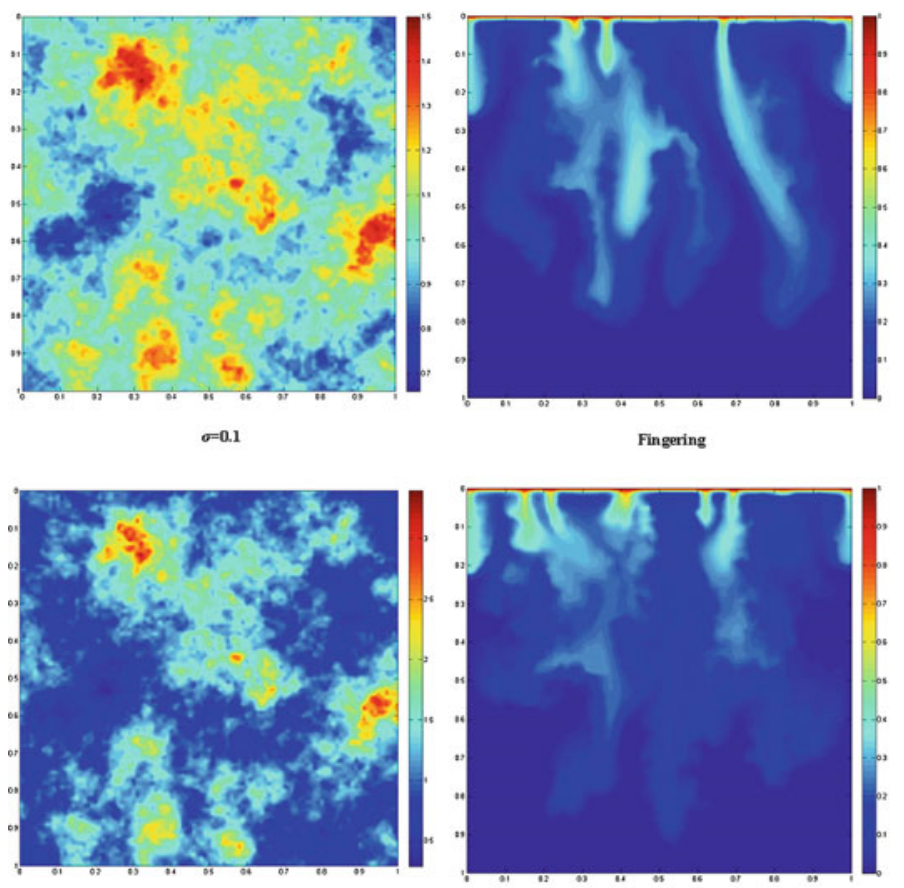

$\sigma=0.3$

Channelling

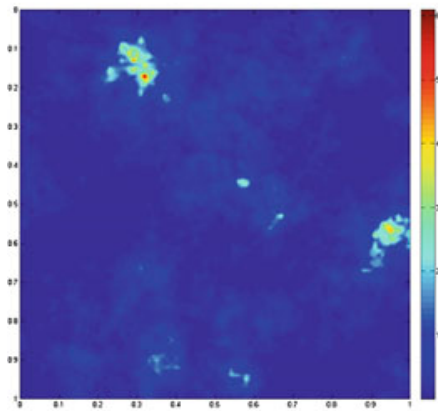

$\sigma=10$

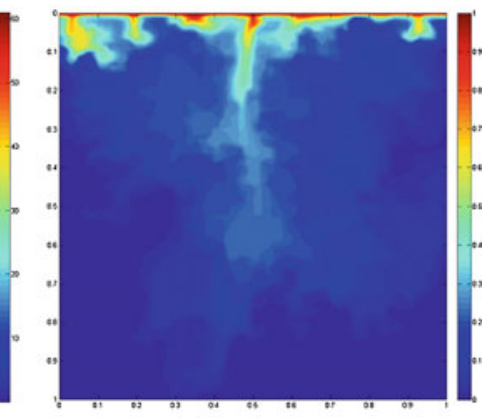

Channelling
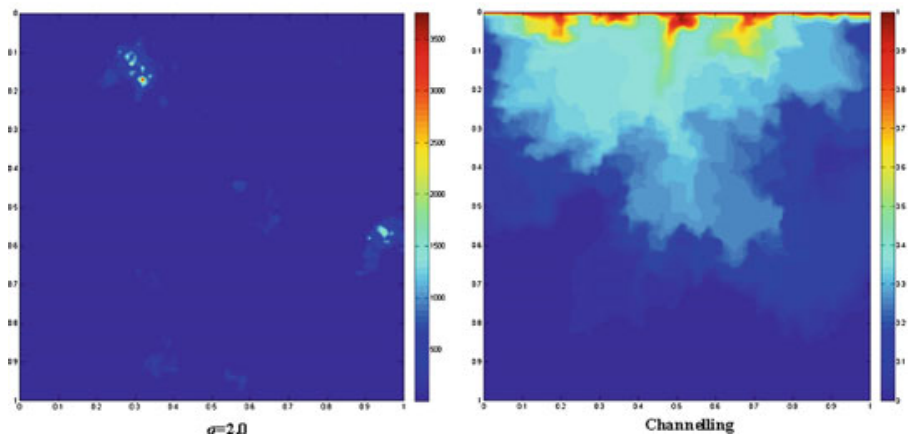

Fig. 7 continued 
Figure 7 shows that the fingering behavior of instabilities occurs for all values of the correlation length $(0.025-0.25)$ at lower value of standard deviation $(\sigma=0.1)$. The channeling behavior of instabilities occurs for all values of the correlation length at standard deviations below $\sigma=0.3$; at a higher standard deviation i.e., 1 and 2 it occurs for a correlation length of 0.25 (Fig. 7). For the correlation length range $(0.025-0.1)$ at higher standard deviations $\sigma=1$ and $\sigma=2$, the instabilities exhibit a dispersive pattern. The dispersive character of the instabilities grows fast at earlier times and become passive at later times due to increase in the dispersive mixing. These findings are consistent with the work of Prasad and Simmons (2003), who observed that an increase in the standard deviation of the log-permeability field caused an increase in instabilities at earlier times and decrease of instabilities at later times.

\subsection{Effect of Correlation Length}

To investigate the effect of the correlation length on the instabilities, we consider the same correlation length in vertical and horizontal directions and set the correlation length to $\lambda=0.025,0.05,0.1$, and 0.25 . Note that each realisation gives a different average permeability where the correlation length becomes comparable to the domain size. Figure 8a, b shows the concentration profiles with log-normal permeability fields for a single realisation with different correlation lengths at standard deviations of 0.3 and 1.0, respectively. The instabilities exhibit a channeling behavior for the correlation lengths examined (0.025-0.25) at a standard deviation of 0.3 . The reason for this behavior is that for larger correlation length (0.25), the log-normal permeability field generates high permeability zones toward which the instabilities tend to move. Also the length scale of permeability is somewhat larger than that of the instabilities formed in the domain. The dispersive pattern occurs at higher standard deviations e.g., for a standard deviation of 1.0 and for a correlation length in the range of $0.025-0.1$ (Fig. $8 \mathrm{a}-\mathrm{c}$ ). This may be due to the fact that for smaller correlation length, the lognormal permeability field leads to local fluctuations in the velocity due to a local change in permeability leading to strong mixing. These observations are consistent with the findings by Prasad and Simmons (2003), who observed that increasing the horizontal correlation length reduction in the extent of fingering.

\subsection{Evolution of the Mixing Length}

Figure 9 shows the variation of the mixing length as a function of the dimensionless time for all realisations of different correlation lengths with standard deviations between 0.3 and 1.0. For a low standard deviation $(\sigma=0.3)$, three regimes can be observed, namely diffusion, convection and second diffusion. In the first regime, the mixing length scales like the square root of time. This indicates that initially diffusion is dominant and convection does not affect the flow significantly. In the second regime, the mixing length varies linearly with time. This shows that the flow is dominated by convection. There is a characteristic time for the onset of convection, i.e., for the transition from the diffusion and convection dominated regimes. In a third regime (second diffusion), the mixing length varies at a rate which is lower than the second regime, but is slightly higher than that of the first regime. This drop in mixing length growth rate is probably due to the presence of non-linear interaction mechanisms such as merging, shielding, tip splitting, and fading.

For a higher standard deviations and for all realisations, only two regimes are observed at lower correlation lengths i.e., between 0.025 and 0.05 . A higher correlation length of 0.1 
(a)
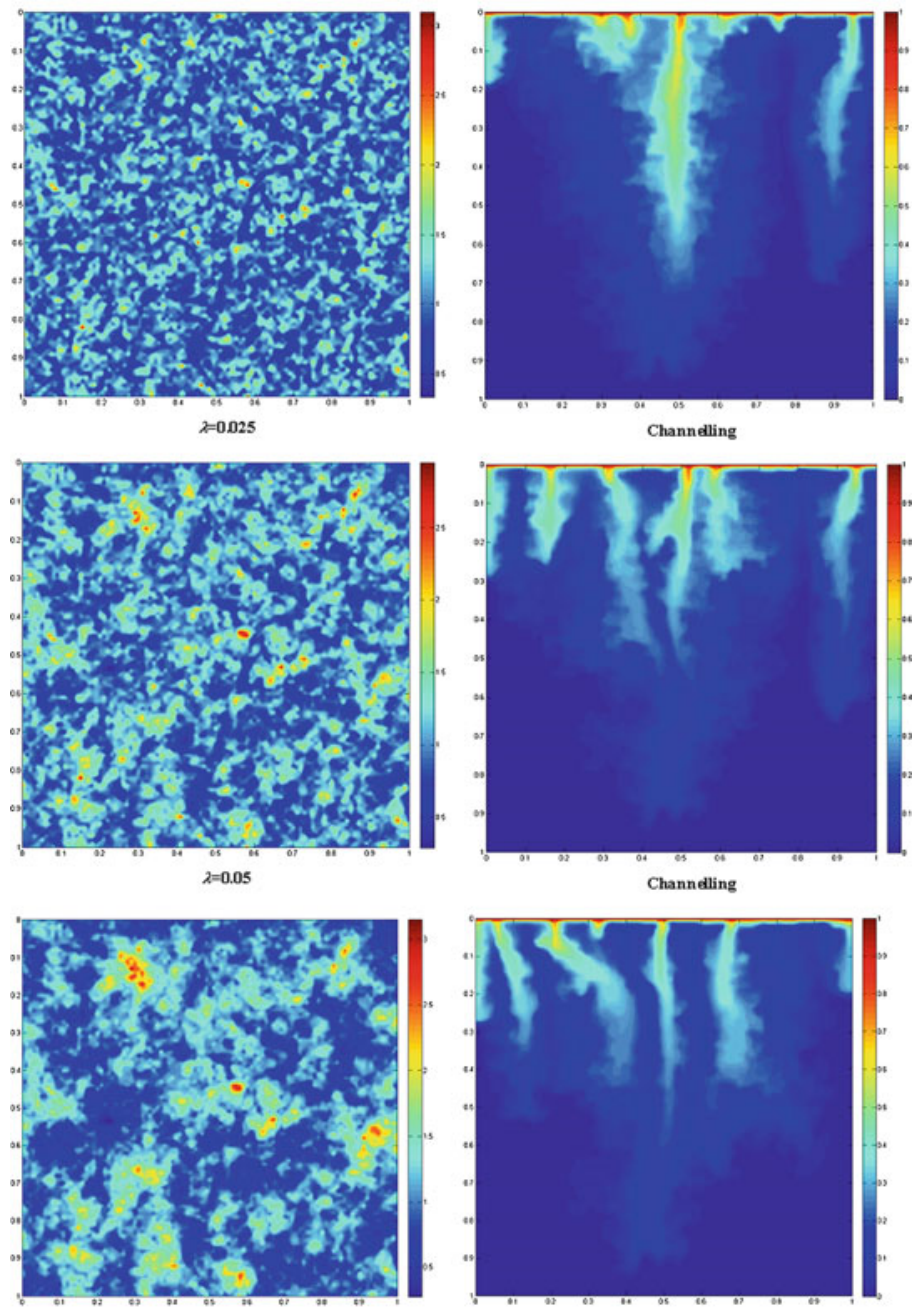

Channelling
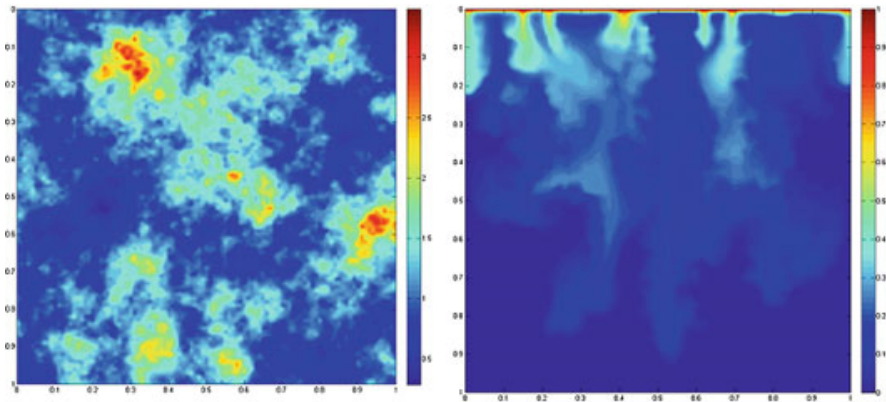

$\lambda=0.2$

Channelling

Fig. 8 Log-normal permeability fields and $\mathrm{CO}_{2}$ concentration profiles of one realisation for Rayleigh number of 10,000 at dimensionless time $t_{d}=0.0005$ with different correlation lengths for standard deviations of (a) 0.3 , (b) 1.0 
(b)
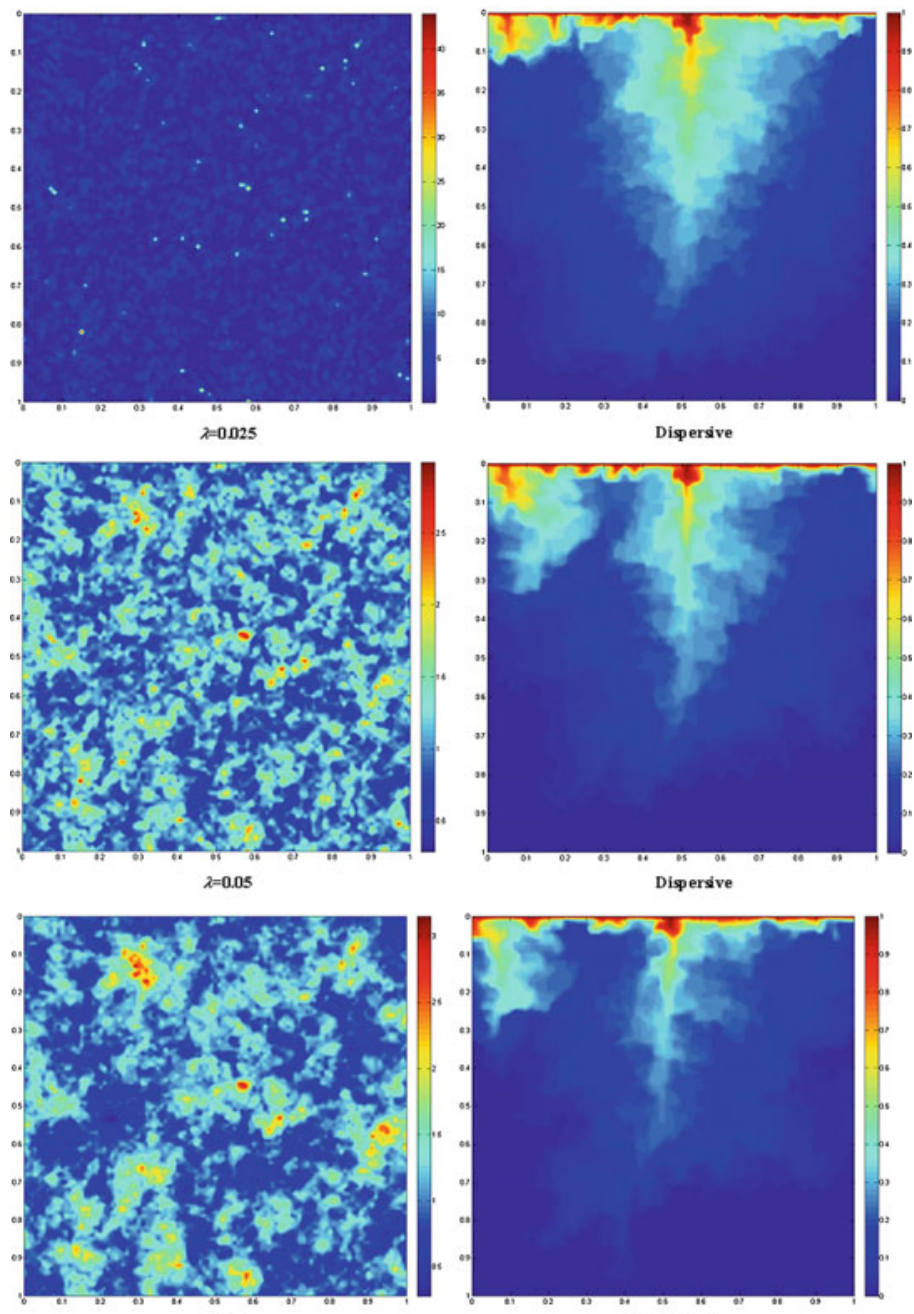

$\lambda=0.1$

Dispersive
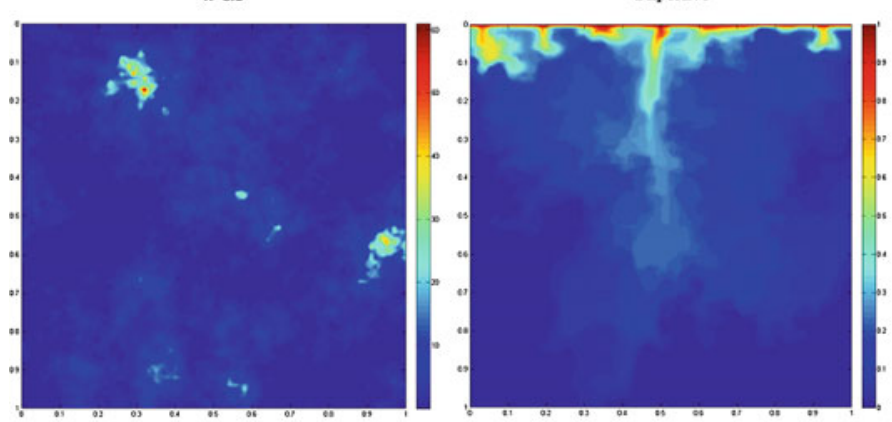

2=02

Channelling

Fig. 8 continued 


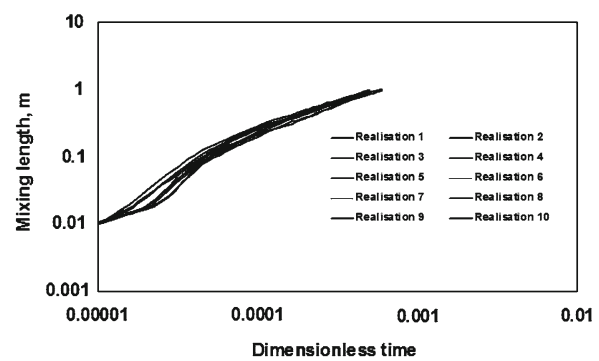

(a) $\sigma=0.3$ and $\lambda=0.025$

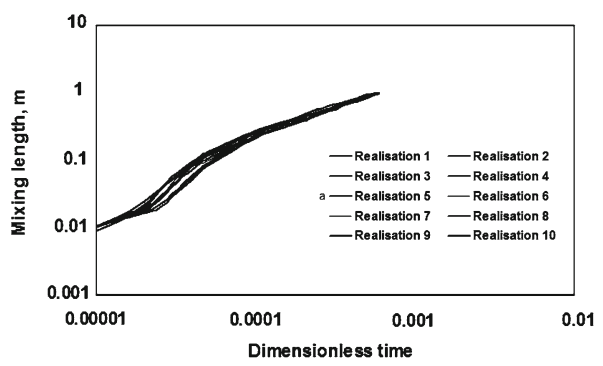

(c) $\sigma=0.3$ and $\lambda=0.1$

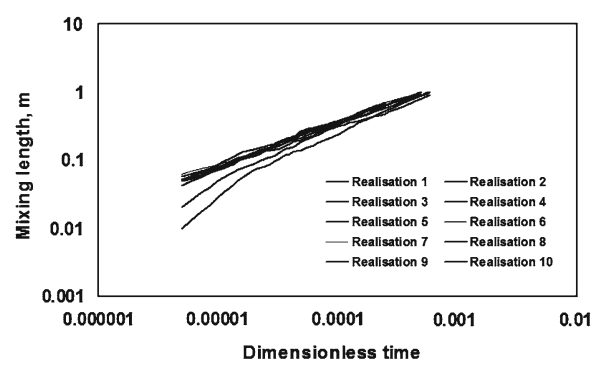

(e) $\sigma=1.0$ and $\lambda=0.025$

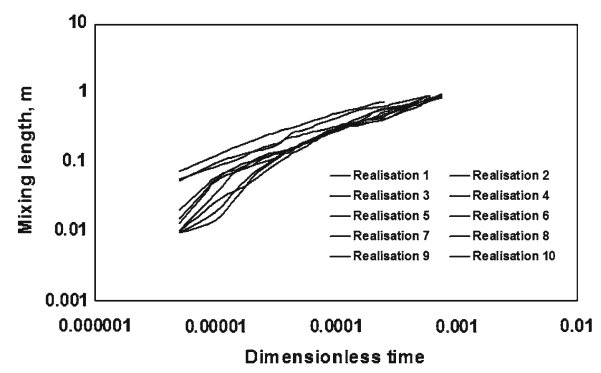

(g) $\sigma=1.0$ and $\lambda=0.1$

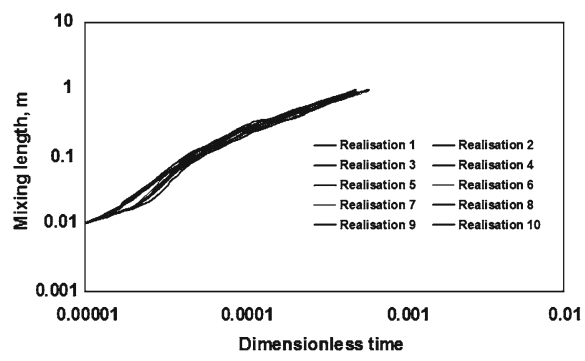

(b) $\sigma=0.3$ and $\lambda=0.05$

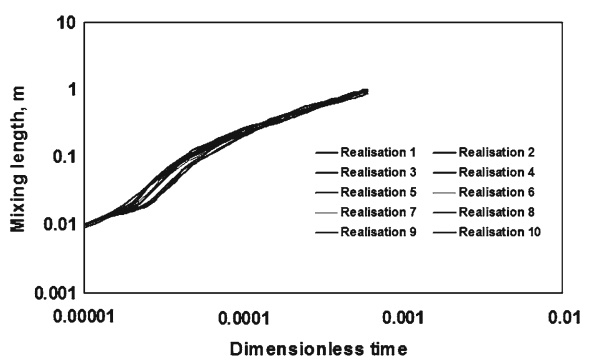

(d) $\sigma=0.3$ and $\lambda=0.25$

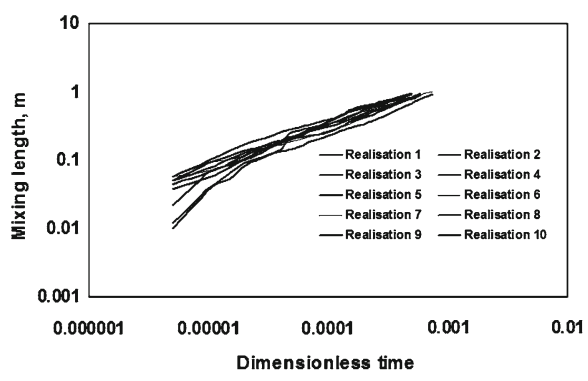

(f) $\sigma=1.0$ and $\lambda=0.05$

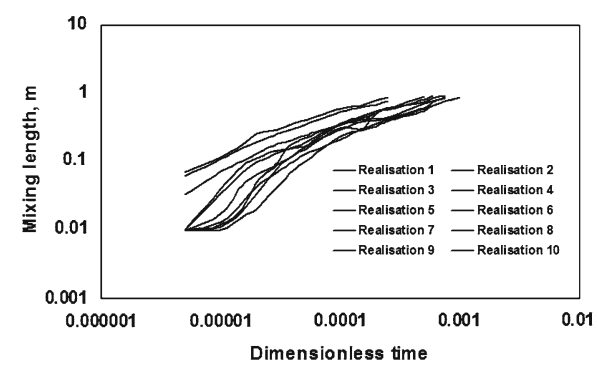

(h) $\sigma=1.0$ and $\lambda=0.25$

Fig. 9 Mixing length variation as a function of dimensionless time for multiple realisations of different correlation lengths with standard deviations of 0.3 and 1.0 

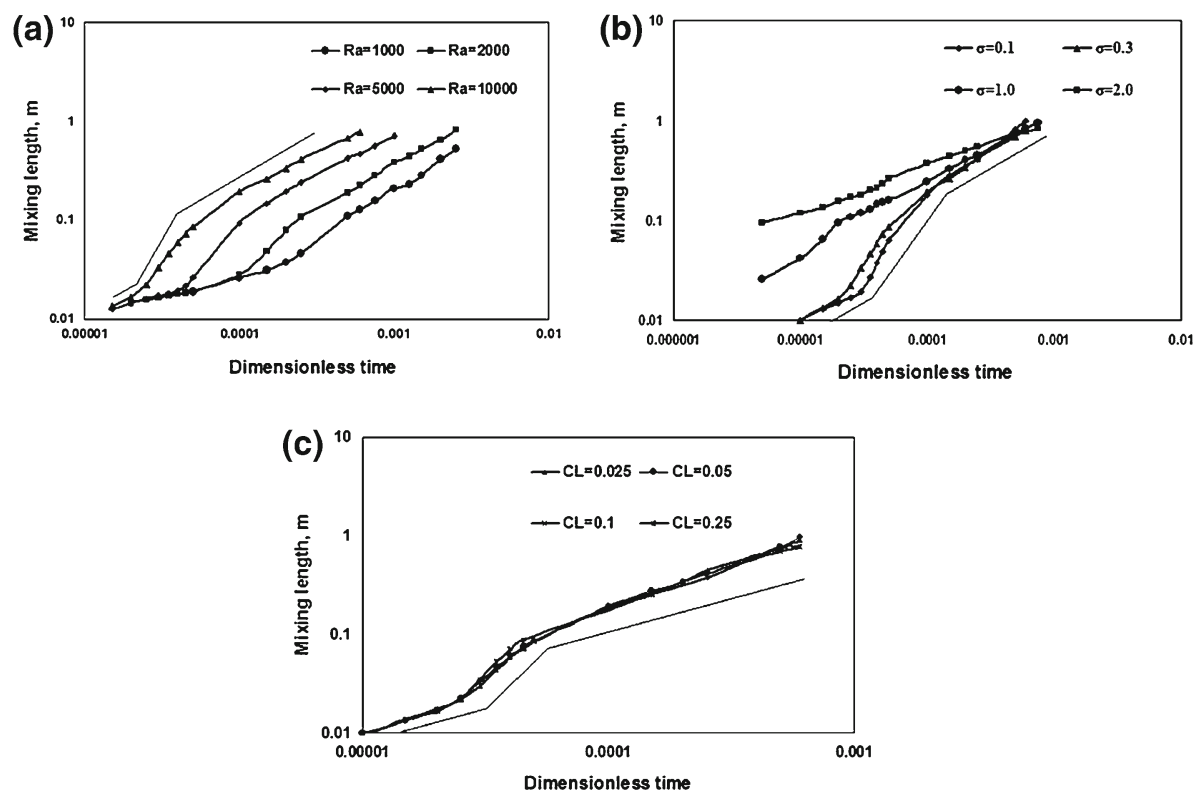

Fig. 10 Mixing length growth with time (a) various Rayleigh numbers, (b) various standard deviations, (c) various correlation lengths

and 0.25 , only a few realisations give three regimes but most of the realisations show only two regimes. These two regimes are similar to the second and third regimes that are obtained earlier (Fig. 9a). The occurrence of only two regimes can be explained by the fact that for high correlation lengths and at earlier times, the growth of the fingers is dominant, which gives an increasing mixing length. At later times, the higher standard deviation promotes lateral dispersive mixing, which leads to slower motion of the most advanced fingers. The data given in the figure also shows that for low standard deviations all realisations have the same mixing length and for high standard deviations there is a large variation of the mixing length. However, for the high standard deviations the mixing length obtained from different realisations show large deviation.

Figure 10a-c shows the variation of the mixing length versus dimensionless time obtained by averaging ten realisations as a function of the Rayleigh number, standard deviation, and correlation length of the log-normal permeability fields. Fig. 10a clearly exhibits the three regimes discussed in Fig. 9a, for various Rayleigh numbers. It can be seen that the transition time from diffusion dominated flow to convection dominated flow decreases as the Rayleigh number increases. Furthermore, it is found similarly to the above graphs that the three mixing length regimes reduce to two regimes when the standard deviation increases from 0.3 to 1.0. For all correlation lengths, the profiles of the mixing length variation show a similar pattern, as in Fig. 10c. Thus, the effect of correlation length on the growth of mixing length is small.

\subsection{Average $\mathrm{CO}_{2}$ Mass Flux}

Figure 11 shows the average $\mathrm{CO}_{2}$ mass flux at the top boundary as a function of the dimensionless time for ten realisations examined by varying the correlation length at a standard 


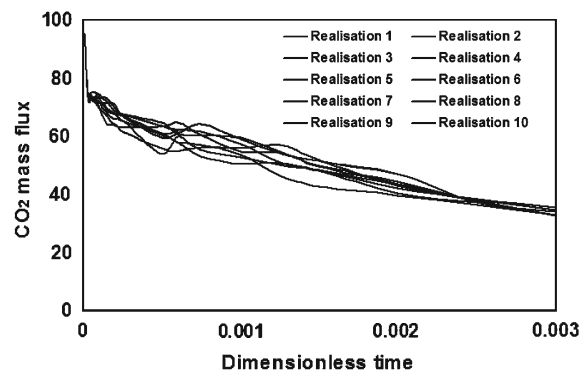

(a) $\sigma=0.3$ and $\lambda=0.025$

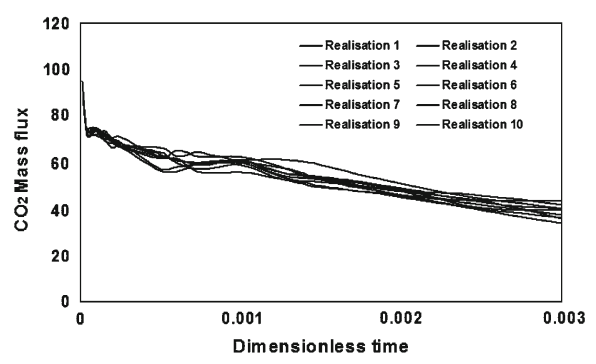

(c) $\sigma=0.3$ and $\lambda=0.1$

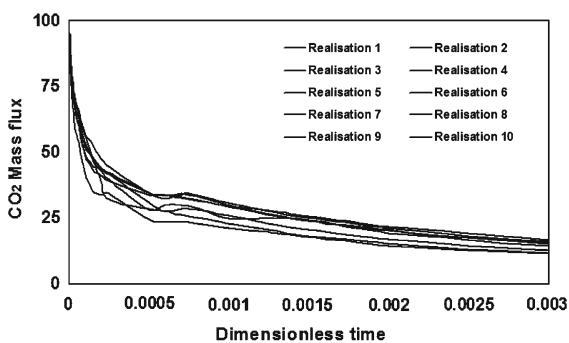

(e) $\sigma=1.0$ and $\lambda=0.025$

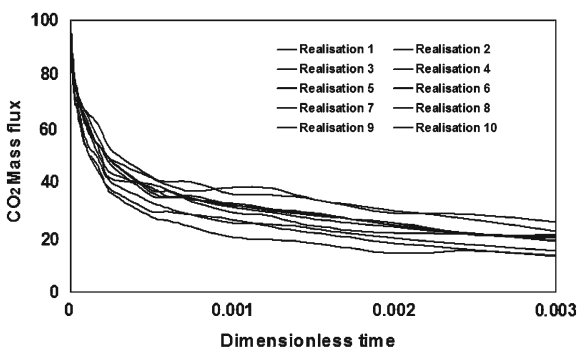

(g) $\sigma=1.0$ and $\lambda=0.1$

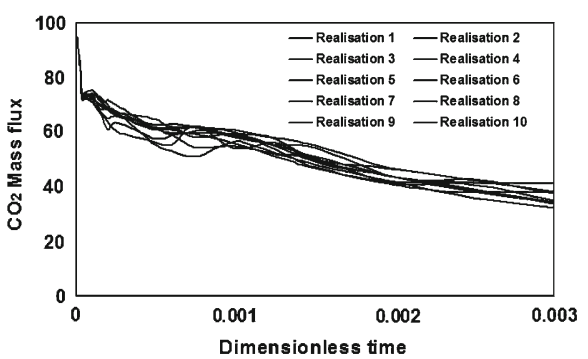

(b) $\sigma=0.3$ and $\lambda=0.05$

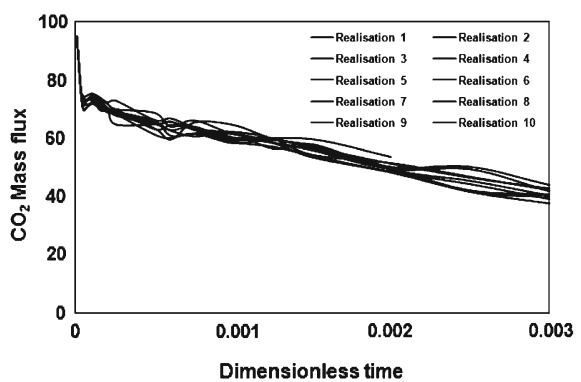

(d) $\sigma=0.3$ and $\lambda=0.25$

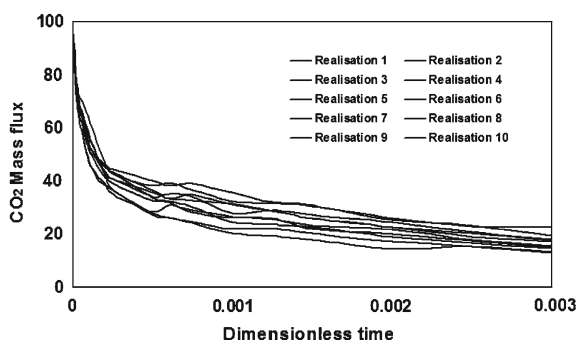

(f) $\sigma=1.0$ and $\lambda=0.025$

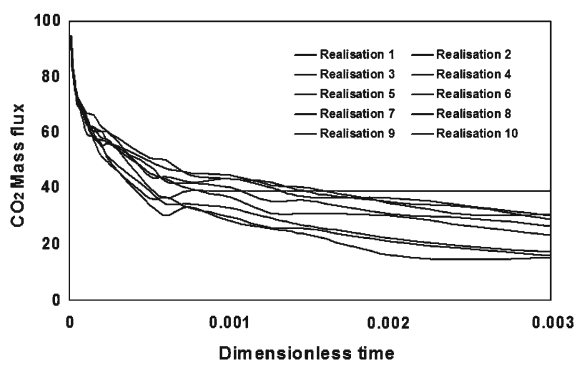

(h) $\sigma=1.0$ and $\lambda=0.1$

Fig. $11 \mathrm{CO}_{2}$ mass flux at top boundary as a function of dimensionless time for multiple realisations of different correlation lengths with standard deviations of 0.3 and 1.0 


\section{(a)}

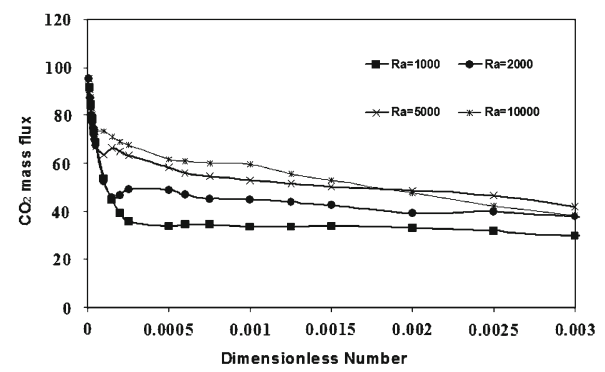

(b)

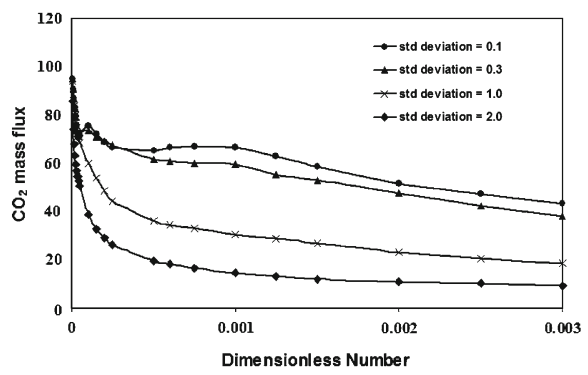

(c)

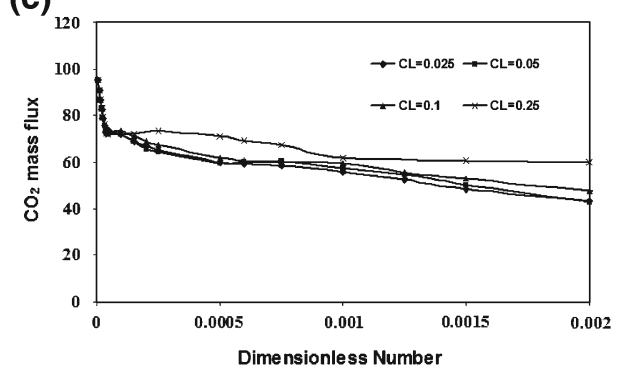

Fig. 12 Plot of average $\mathrm{CO}_{2}$ mass flux at the top boundary versus dimensionless time (a) various Rayleigh numbers, (b) various standard deviations, (c) various correlation lengths

deviation of 0.3 and 1.0, respectively. For all realisations, the $\mathrm{CO}_{2}$ mass flux has a rapidly decreasing trend at the early times and after slight fluctuations it decreases slowly toward a constant value. This can be explained by observing that at early times, the mass transport of $\mathrm{CO}_{2}$ from the top boundary is only controlled by diffusion. In cases where the $\mathrm{CO}_{2}$ mass flux fluctuates, the first peak represents the onset of convection. The constant value of the average mass flux may be explained by the fact that the domain is nearly saturated with dissolved $\mathrm{CO}_{2}$ after the fingers reach the bottom boundary. It is also shown from the figure that for lower standard deviations the average flux of $\mathrm{CO}_{2}$ trend is the same for all realisations. However, for larger standard deviations the mass flux obtained from different realisations shows broader variations.

Figure $12 \mathrm{a}-\mathrm{c}$ shows the variation of the average $\mathrm{CO}_{2}$ mass flux from the top boundary obtained by averaging ten realisations, as a function of the Rayleigh number, standard deviation and correlation length of the log-normal permeability field. First, the $\mathrm{CO}_{2}$ mass flux decreases quickly at earlier times for all Rayleigh numbers, increases slightly and then levels off to a plateau (Fig. 12a). It is also shown that the time at which the $\mathrm{CO}_{2}$ mass flux increases, diminishes for higher Rayleigh numbers. Figure $12 \mathrm{~b}$ shows the average $\mathrm{CO}_{2}$ mass flux at the top boundary as a function of dimensionless time for different standard deviations. For the lower standard deviation of 0.3 and 1.0, the average $\mathrm{CO}_{2}$ mass flux as a function of dimensionless time decreases quickly at early times. Subsequently increases before it decreases again. For the higher standard deviation of 1 and 2, the average $\mathrm{CO}_{2}$ mass flux decreases with time and then reaches a stabilized value of the mass flux at later times. This shows that the transport of mass is mainly controlled by dispersive mixing. The effect of the correlation length on the average $\mathrm{CO}_{2}$ mass flux is shown in Fig. 12c. We observe that there is little variation in the calculation of the average $\mathrm{CO}_{2}$ mass flux for different correlation lengths. In 
(a)

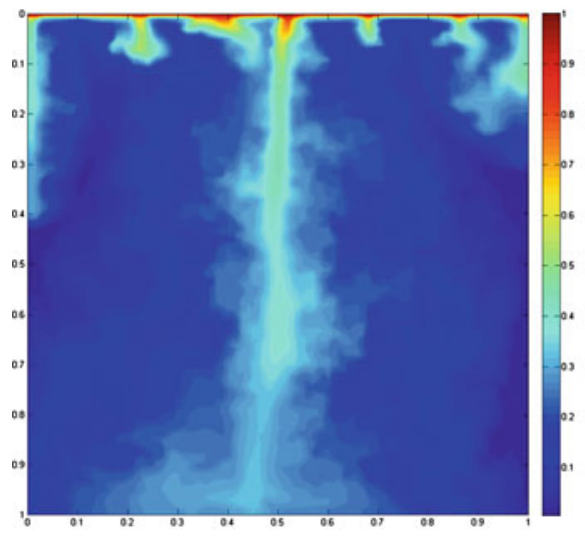

(b)

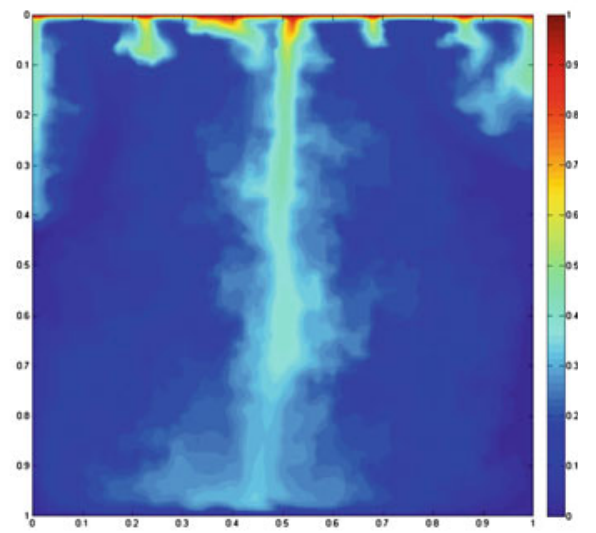

(c)

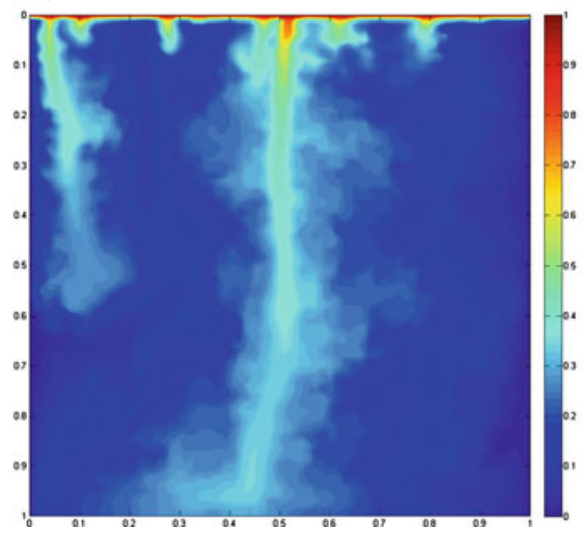

Fig. 13 Effect of various boundary conditions on the $\mathrm{CO}_{2}$ concentration profiles of one realisation $(\lambda=0.1$ and $\sigma=0.3$ ) at the dimensionless time of $t_{d}=0.001$ (a) no-flow conditions for all boundaries, (b) no-flow conditions for lateral boundaries and constant bottom boundary, (c) periodic condition for lateral boundaries and no-flow at bottom boundary

general, it can be concluded that the effect of correlation length on the average $\mathrm{CO}_{2}$ mass flux is small.

\subsection{Effect of the Boundary Conditions}

A series of simulations were carried out to test the influence of the boundary conditions on $\mathrm{CO}_{2}$ concentration profiles. The boundary conditions examined are: (a) no-flow conditions for lateral and bottom boundaries, (b) periodic conditions for lateral boundaries and no-flow at bottom boundary, and (c) no-flow conditions for the lateral boundaries and zero concentration at bottom boundary. The concentration profiles and average $\mathrm{CO}_{2}$ mass flux at the top boundary obtained from these simulations are shown in Figs. 13 and 14, respectively. The concentration profiles are rather similar for the different boundary conditions tested, while there is considerable difference in concentration profiles at the lateral posi- 


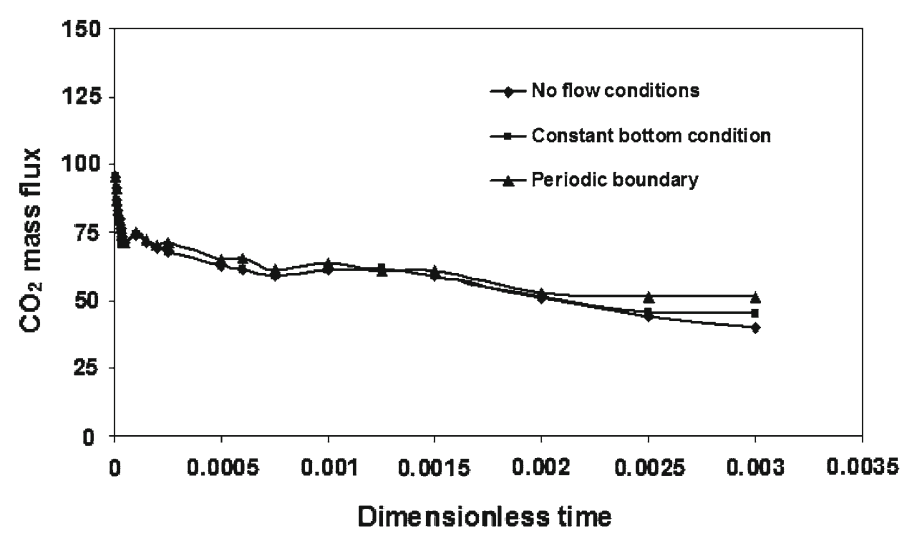

Fig. 14 Plot of average $\mathrm{CO}_{2}$ mass flux at the top boundary versus dimensionless time for Rayleigh number of 10,000 with various boundary conditions

tions for periodic boundary conditions. For the no-flow boundary condition, a few gravity fingers are shown to develop along the lateral boundaries, but the extent of these fingers is less than the extent of the fingers in the interior of the region. For the periodic boundary condition, the $\mathrm{CO}_{2}$ concentration allows lateral flow, which tends to spread the $\mathrm{CO}_{2}$ plume. The constant concentration condition $(c=0)$ at the bottom boundary gives an unrealistic pattern at the bottom of the domain. This may be due to the fact that $\mathrm{CO}_{2}$ is lost through diffusion across the bottom boundary. Figure 14 reveals that the $\mathrm{CO}_{2}$ mass flux at the top boundary with time has nearly the same trend for all boundary conditions. At later times the average $\mathrm{CO}_{2}$ mass flux is slightly higher for a periodic condition compared to other boundary conditions. Most probably the $\mathrm{CO}_{2}$ plume spreads more when lateral flow is admitted.

\subsection{Effect of Height and Width of Domain}

To investigate the effect of width and height of the flow domain on the development of instabilities particularly at longer times, we plot the $\mathrm{CO}_{2}$ concentration profiles at the time when the fingers reach the bottom boundary for the different values of height and width of the domain. The height and width of the domain examined in the present study are 1.0, 2.0, and 4.0. The concentration profiles of one of the realisations (correlation length of 0.1 , standard deviation of 0.3 , and Rayleigh number of 10,000) for various height and width of domain are shown in Figs. 15 and 16, respectively. It can be observed from Fig. 15 that a few large-scale dominant fingers are formed due to merging of small-scale fingers and reach the bottom boundary of the domain. The number of the large-scale dominant fingers decreases as the height of the domain increases. The effect of the width of the domain on instabilities shows that the interactions between fingers reduce, and the fingers move independently to reach the bottom boundary. This trend is more pronounced when the width of domain increases. It is also observed that the time when fingers reach the bottom boundary increases as the width of the domain increases. 
(a)

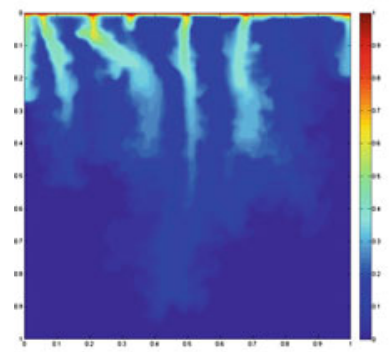

(b)

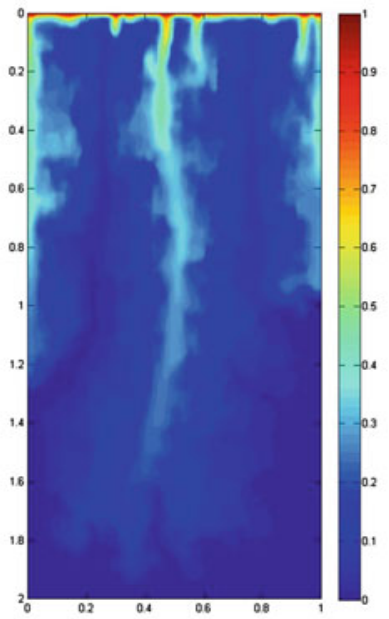

(c)

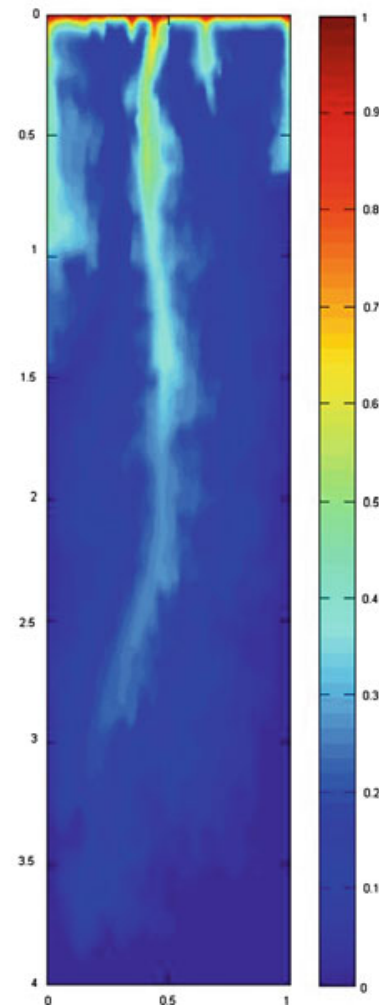

Fig. 15 Effect of the height of the domain on $\mathrm{CO}_{2}$ concentration profiles with log-normal permeability fields of one realisation $(\lambda=0.1$ and $\sigma=0.3)$ for Rayleigh number of 10,000 . (a) $H=1$ at $t_{d}=0.0005$, (b) $H=2$ at $t_{d}=0.00125$, (c) $H=4$ at $t_{d}=0.0025$

\section{Conclusions}

Density-driven natural convection in heterogeneous porous media, where a $\mathrm{CO}_{2}$-rich gaseous phase overlays a liquid saturated medium, was investigated through numerical modeling. Ten realisations of log-normal permeability fields generated by the sequential gaussian simulation (SGSIM) method were used to do the numerical analyses. The convective motion was examined in terms of concentration profiles, variation of mixing length, and the average $\mathrm{CO}_{2}$ mass flux at the top boundary as a function of the Rayleigh numbers, the standard deviation of heterogeneity characteristic, and the correlation length of the permeability distributions. The following main conclusions can be drawn from this study:

- For various correlation lengths at lower standard deviation, a gravity-induced fingering pattern was observed; at moderate standard deviations of the log-normal permeability field, the flow exhibits a channel pattern, which is controlled by the permeability field. At higher standard deviations the flow was dispersive when the correlation length is small, whereas a high correlation lengths, the flow was characterized by a channel pattern.

- For various Rayleigh numbers, three different regimes were deduced from the plot of the mixing length variation with dimensionless time. In the first regime, the mass transfer 


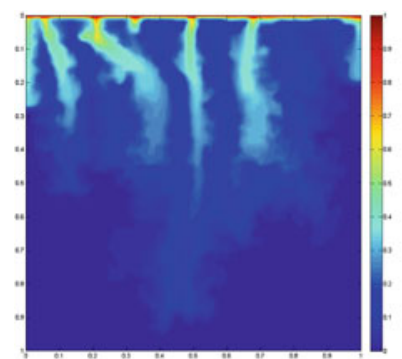

(a)

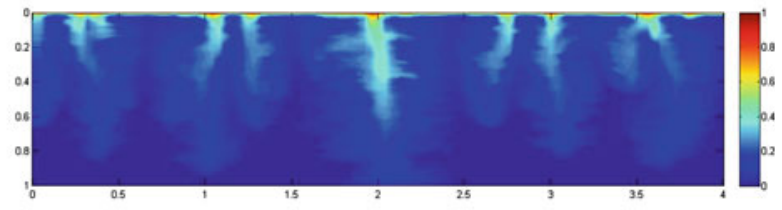

(b)

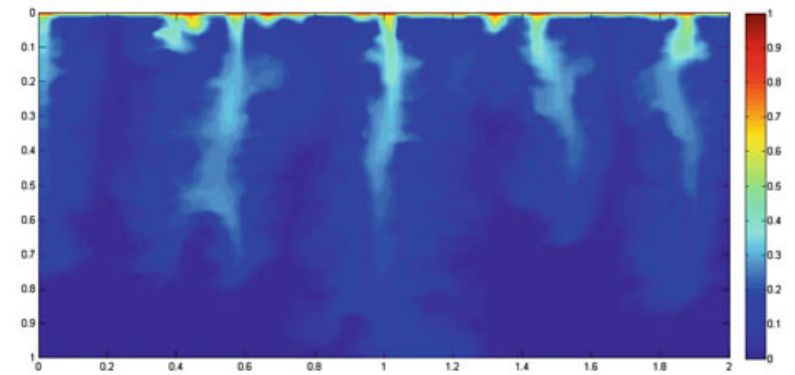

(c)

Fig. 16 Effect of the width of the domain on $\mathrm{CO}_{2}$ concentration profiles with log-normal permeability fields of one realisation $(\lambda=0.1$ and $\sigma=0.3)$ for Rayleigh number of 10,000. (a) $w=1$ at $t_{d}=0.0006,(\mathbf{b}) w=2$ at $t_{d}=0.00075$, (c) $w=4$ at $t_{d}=0.0001$

is dominated by diffusion; in the second regime convection starts and mass transfer is controlled by convective mixing and in the third regime, the rate of mixing length growth is lower than that in the second regime, but is nearly equal to that of the first regime. For higher standard deviations, there are only two regimes, i.e., the first diffusion dominated regime is absent. The mixing length is only weakly dependent on the correlation length.

- For all values of the Rayleigh numbers, at first the average $\mathrm{CO}_{2}$ mass flux decreases quickly at earlier times, increases slightly at later times and then levels off to a plateau. The time at which the $\mathrm{CO}_{2}$ mass flux increases, diminishes for higher Rayleigh numbers. It was found that at early times and for the lower standard deviation, the average $\mathrm{CO}_{2}$ mass flux decreases rapidly; however, for later times it increases and then decreases. For higher standard deviations, the average $\mathrm{CO}_{2}$ mass flux decreases with time and reaches a constant value at later times. We found that the effect of the correlation length on the average $\mathrm{CO}_{2}$ mass flux is small.

- The effect of boundary condition shows significant changes in $\mathrm{CO}_{2}$ concentration profile while a little change in $\mathrm{CO}_{2}$ mass flux at later times. With increasing the height of the domain, a few large-scale dominant fingers are formed due to interactions between fingers. With increasing the width of the domain, fingers move independently without the interact each other.

Acknowledgments This study was partly done as part of the CATO II programme. The authors are grateful to the Dutch Ministry of Economic Affaires and the sponsoring companies for funding. The authors are also grateful to the anonymous refereed whose comments and suggestions have help improve manuscript. 
Open Access This article is distributed under the terms of the Creative Commons Attribution License which permits any use, distribution, and reproduction in any medium, provided the original author(s) and the source are credited.

\section{References}

Barlebo, H.C., Hill, M.C., Rosbjer, D.: Investigating the macrodispersion experiment (MADE) site in Columbus, Mississippi, using a three-dimensional inverse flow and transport model. Water Resour. Res. 40, W04211 (2004)

Bear, J.: Dynamics of Fluids in Porous Media. Dover Publications, New York (1972)

Bryant, S.L., Lakshminarasimhan, S., Pope, G.A.: Buoyancy-dominated multiphase flow and its effect on geological sequestration of $\mathrm{CO}_{2}$. SPE J. 13, 447-454 (2008)

Deutsch, C.V., Journel, A.G.: GSLIB Geostatistical Software Library and Users Guide. Oxford University Press, New York (1992)

Elder, J.W.: Transient convection in a porous medium. J. Fluid Mech. 27, 609-623 (1967)

Ennis-King, J., Paterson, L.: Role of convective mixing in the long-term storage of carbon dioxide in deep saline formations. SPE 84344, 1-12 (2005)

Ennis-King, J., Paterson, L.: Rate of dissolution due to convective mixing in the underground storage of carbon dioxide. In: Gale, J., Kaya, Y. (eds.) Greenhouse Gas Control Technologies, vol. 1, pp. 507-510. Elsevier, Amsterdam (2003)

Farajzadeh, R., Salimi, H., Zitha, P.L.J., Bruining, J.: Numerical simulation of density-driven natural convection in porous media with application for $\mathrm{CO}_{2}$ injection projects. Int. J. Heat Mass Transf. 50, 50545064 (2007)

Farajzadeh, R., Farshbaf Zinati, F., Zitha, P.L.J., Bruining, J.: Density driven natural convection in layered and anisotropic porous media. In: Proceeding of the 11th European Conference on Mathematics in Oil Recovery (ECMOR X1), Norway (2008)

Farajzadeh, R., Ranganathan, P., Zitha, P.L.J., Bruining, J.: The effect of heterogeneity on the character of density-driven natural convection of $\mathrm{CO}_{2}$ overlying a brine layer. Adv. Water Resour. 34, 327-339 (2011)

Firoozabadi, A., Cheng, P.: Prospects for subsurface $\mathrm{CO}_{2}$ sequestration. AIChE J. 56, 1398-1405 (2010)

Gebhart, B., Jaluria, Y., Mahajan, R.L., Sammakia, B.: Buoyancy-Induced Flows and Transport. Hemisphere, Harper and Row, New York (1988)

Ghesmat, K., Azaiez, J.: Viscous fingering instability in porous media: Effect of anisotropic velocity-dependent dispersion tensor. Transp. Porous Med. 73, 297-318 (2008)

Green, C., Ennis-King, J.: Effect of vertical heterogeneity on long-term migration of $\mathrm{CO}_{2}$ in saline formations. Transp. Porous Med. 82, 31-47 (2010)

Guceri, S., Farouk, B.: Numerical solutions in laminar and turbulent natural convection. In: Kakac, S., Aung, W., Viskanta, R. (eds.) Natural Convection, Fundamentals and Applications, pp. 615-655. Hemisphere, Bristol, PA (1985)

Han, W.S., Lee, S., Lu, C., McPherson, B.J.: Effect of permeability on $\mathrm{CO}_{2}$ trapping mechanism and buoyancydriven $\mathrm{CO}_{2}$ migration in saline formations. Water Resour. Res. 46, 1-20 (2010)

Hassanzadeh, H., Pooladi-Darvish, M., Keith, D.W.: Scaling behavior of convective mixing, with application to geological storage of $\mathrm{CO}_{2}$ storage. AIChE J. 55, 1121-1131 (2007)

Hidalgo, J.J., Carrera, J.: Effect of dispersion on the onset of convection during $\mathrm{CO}_{2}$ sequestration. J. Fluid Mech. 640, 441-452 (2009)

Kneafsey, T.J., Pruess, K.: Laboratory flow experiments for visualizing carbon dioxide-induced, density-driven brine convection. Transp. Porous Med. 82, 123-139 (2010)

LeBlanc, D.R., Garabedian, S.P., Hess, K.M., Gelhar, L.W., Quadri, R.D., Stollenwerk, K.G., Wood, W.W.: Large-scale natural gradient tracer test in sand and gravel, Cape Cod, Massachusetts. 1. Experimental design and observed tracer movement. Water Resour. Res. 27, 895-910 (1991)

Li, Z., Dong, M., Shirif, E.: Transient natural convection induced by gas diffusion in liquid-saturated vertical porous columns. Ind. Eng. Chem. Res. 45, 3311-3319 (2006)

Lindeberg, E., Bergmo, P.: The long-term fate of $\mathrm{CO}_{2}$ injected into an aquifer. In: Gale, J., Kaya, Y. (eds.) Greenhouse Gas Control Technologies, vol. 1, pp. 489-494. Elsevier, Amsterdam (2003)

Malkovsky, V.I., Pek, A.A.: Onset conditions of free thermal convection of a single-phase fluid in a horizontal porous layer with depth-dependent permeability. Izvestiya Phys. Solid Earth 35, 990-994 (1999)

McKibbin, R., O'Sullivan, M.J.: Onset of convection in a layered porous medium heated from below. J. Fluid Mech. 96, 375-393 (1980)

Nield, D.A., Bejan, A.: Convection in Porous Media. Springer, New York (1999) 
Nield, D.A., Simmons, C.T.: A discussion on the effect of heterogeneity on the onset of convection in a porous medium. Transp. Porous Med. 68, 413-421 (2007)

Nield, D.A., Kuznetsov, A.V., Simmons, C.T.: The effect of strong heterogeneity on the onset of convection in a porous medium: 2D/3D Localization and spatially correlated random permeability fields. Transp. Porous Med. 83, 465-477 (2010)

Pau, G.S.H., Bell, J.B., Pruess, K., Almgren, A.S., Lijewski, M.J., Zhang, K.: High resolution simulation and characterization of density-driven flow in $\mathrm{CO}_{2}$ storage in saline aquifers. Adv. Water Resour. 33, 443-455 (2010)

Prasad, A., Simmons, C.T.: Unstable density-driven flow in heterogeneous porous media: a stochastic study of the Elder [1967b] "short heater" problem. Water Resour. Res. 39, 1-21 (2003)

Pruess, K., Zhang, K.: Numerical Modeling Studies of the dissolution-diffusion-convection process during $\mathrm{CO}_{2}$ storage in saline aquifers. Lawrence Berkeley Laboratory Report LBNL-1243E, Berkeley, CA (2008)

Rapaka, S., Chen, S., Pawar, R.J., Stauffer, P.H., Zhang, D.: Nonmodal growth of perturbations in densitydriven convection in porous media. J. Fluid Mech. 609, 285-303 (2008)

Rapaka, S., Chen, S., Pawar, R.J., Stauffer, P.H., Zhang, D., Chen, S.: Onset of convection over a transient base-state in anisotropic and layered porous media. J. Fluid Mech. 641, 227-244 (2009)

Riaz, A., Hesse, A., Tchelepi, M., Orr, F.M.: Onset of convection in a gravitationally unstable diffusive boundary layer in porous medium. J. Fluid Mech. 548, 87-111 (2006)

Rogerson, A., Meiburg, E.: Numerical simulation of miscible displacement processes in porous media flows under gravity. Phys. Fluids A 5, 2644-2660 (1993)

Schincariol, R.A., Schwartz, F.W., Mendoza, C.A.: Instabilities in variable density flows: stability and sensitivity analyses for homogeneous and heterogeneous media. Water Resour. Res. 33, 31-41 (1997)

Shinozuka, M., Jan, C.M.: Digital simulation of random processes and its applications. J. Sound Vib. 25, 357-368 (1972)

Simmons, C.T., Narayan, K.A., Wooding, R.A.: On a test case for density-dependent groundwater flow and solute transport models: the salt lake problem. Water Resour. Res. 35, 3607-3620 (1999)

Simmons, C.T., Fenstemaker, T.R., Sharp, J.M.: Variable-density groundwater flow and solute transport in heterogeneous porous media: approaches, resolutions and future challenges. J. Contam. Hydrol. 52, 245275 (2001)

Sorbie, K.S., Farag, F., Pickup, K.S., Ringrose, P.S., Jensen, J.L.: Flow regimes in miscible displacements in heterogeneous correlated random fields. SPE Adv. Technol. Ser. 2, $78-87$ (1994)

Waggoner, J.R., Castillo, J.L., Lake, L.W.: Simulation of EOR processes in stochastically generated permeable media. Soc. Pet. Eng. Form. Eval. 7, 173-180 (1992)

Welty, C., Gelhar, L.W.: Stochastic analysis of the effects of fluid density and viscosity variability on macrodispersion in heterogeneous porous media. Water Resour. Res. 27, 2061-2075 (1991)

Welty, C., Gelhar, L.W.: Simulation of large-scale transport of variable density and viscosity fluids using a stochastic mean model. Water Resour. Res. 28, 815-827 (1992)

Zimmerman, W.B., Homsy, G.M.: Viscous fingering in miscible displacement: unification of effects of viscosity contrast, anisotropic dispersion, and velocity dependence of dispersion on nonlinear finger propagation. Phys. Fluids A 4, 348-2359 (1992) 